\title{
EXPERIMENTAL MODAL ANALYSIS OF STRUCTURAL SYSTEMS BY USING THE FAST RELAXED VECTOR FITTING METHOD
}

\author{
Marco Civera $^{a}$, Giulia Calamai ${ }^{\mathrm{b}}$, and Luca Zanotti Fragonara ${ }^{\mathrm{c}}$ \\ ${ }^{\text {a }}$ Department of Mechanical and Aerospace Engineering, Politecnico di Torino, 10129 \\ Turin, Italy \\ ${ }^{\mathrm{b}}$ Department of Structural, Building and Geotechnical Engineering, Politecnico di \\ Torino, 10129 Turin, Italy \\ ${ }^{\mathrm{c}}$ Centre for Autonomous and Cyber-Physical Systems, Cranfield University, Cranfield, \\ Bedford MK43 0AL, UK
}

\begin{abstract}
System identification (SI) techniques can be used to identify the dynamic parameters of mechanical systems and civil infrastructures. The aim is to rapidly and consistently model the object of interest, in a quantitative and principled manner. This is also useful in establishing the capacity of a structure to serve its purpose, thus as a tool for Structural Health Monitoring (SHM). In this context, input-output SI techniques allow precise and robust identification regardless of the actual input. However, one of the most popular and widely used approaches, the Rational Fraction Polynomial (RFP) Method, has several drawbacks. The fitting problem is nonlinear and generally non-convex, with many local minima; even if linearised via weighting, it can become severely ill-conditioned. Here, a novel proposal for the broadband macro-modelling of structures in the frequency domain with several output and/or input channels is presented. A variant of the Vector Fitting approach, the Fast Relaxed Vector Fitting (FRVF), applied so far in the literature only for the identification of electrical circuits, is translated and adapted to serve as a technique for structural SI and compared with other traditional techniques. A study about the robustness of FRVF with respect to noise is carried out on a numerical system. Finally, the method is applied to two experimental case studies: a scaled-model of a High-Aspect-Ratio (HAR) wing and the well-known benchmark problem of the three-storey frame of Los Alamos laboratories. Promising results were achieved in terms of accuracy and computational performance.
\end{abstract}

Keywords: System Identification; Modal Analysis; Vector Fitting; Frequency Response Function; Structural Health Monitoring. 


\section{Introduction}

In the last decades, the use of vibration-based structural health monitoring has been constantly increasing for both existing and new structures, especially leveraging frequency-domain techniques and Experimental Modal Analysis (EMA)[1]. The aims of structural System Identification (SI) are innumerable: evaluating the structural conditions of civil infrastructures, estimating the payload vibrational constraints for launch vehicles, or guessing the dynamic behaviour of complicated rotating machinery or standing structures are only a few of the classic applications. In all cases, the final goal is to define an efficient model equivalent to the provided frequency response samples. For this reason, the research of new methods has become very important. A variety of approaches has been proposed in the last decades, especially resorting to statistical and Bayesian methodologies [2]. Nevertheless, current procedures are still harmed by severe limitations, such as high computational cost, sensitiveness to measurement noise, etc [3].

This work focuses on the implementation of a surrogate modelling algorithm in the frequency domain, known as the Vector Fitting (VF), to identify the properties of a mechanical system. The concept has been firstly developed in the field of electronic engineering [4], where it was initially introduced for the surrogate modelling of large multiport circuits [5]. Since its introduction, Vector Fitting has gained much popularity due to its simplicity, robustness and higher performance and has even been applied in the time domain [6,7]. Specifically, a Fast and Relaxed variant of VF (FRVF) is here implemented. Employing an additional real-valued free parameter and by exploiting the QR decomposition [8,9] for faster calculation, this improved version allows to reach the same results in a more computationally efficient way. This implementation of VF for mechanical systems has been then applied to several numerical and experimental case studies of interest. The advantages of VF and FRVF with respect to similar and related macromodeling techniques, which will be detailed in the following Sections, are wellknown in its field of first application [10] and will surely be appreciated in these new ambits of EMA and modal identification-based Structural Health Monitoring (SHM), for which structural System Identification (SI) is of the utmost importance [11].

The rest of this paper is organised as follow. To begin with, the theoretical background of VF is reported and explained in Section 1. A comparison with closely related strategies and algorithms is presented and the objective advantages of the method are highlighted. In Section 2, the proposed method is applied to numerical case studies to validate the new implementation. Its effectiveness is verified on simulated data with noise artificially added to the input, the output, or both. Hence, the influence of the noise level on the performance of the algorithm is investigated and discussed. Sections 3 reports a preliminary experimental study on a High-Aspect-Ratio (HAR), highly flexible wing prototype. Section 4 describes an application to a common benchmark case study. The confidence of the results has been estimated by using modal criteria, such as Modal Assurance Criterion (MAC), stabilisation and clustering diagrams, and statistical relations, allowing a direct comparison with the values from Literature. The dissertation ends with the Conclusions, which summarise the key findings of this research. 


\section{Theoretical Background.}

Vector Fitting (VF) is an iterative rational approximation technique allowing to estimate the poles and residues of an equivalent rational function by fitting raw frequency samples, typically measured experimentally. The optimisation process is relaxed to a weighted linear Least Squares (LS) problem, which is reformulated and solved iteratively until convergence.

VF is an input-output SI approach, relying on the simple concept that an electrical, mechanical, or hydraulic system can be modelled as the mathematical operator between an excitation signal and the corresponding response signal. In the frequency domain, this can be stated as

$$
Y(\omega)=H(\omega) X(\omega)
$$

where $Y(\omega)$ and $X(\omega)$ are, respectively, the Fourier transforms of the output and the input signals. The same concept can be switched in the time domain without any conceptual limit; it is only more convenient here to express everything as a function of $\omega=2 \pi f$, with $f$ indicating the frequency. It is needed to remind that the use of the Fourier Transform implicitly assumes the linearity of the system under investigation; nevertheless, it is well known that slightly nonlinear mechanisms can be successfully linearised with limited error [12]. The stationarity of the system properties is also assumed here. The system under analysis, $H(\omega)$, can be therefore expressed in terms of its poles and residues.

\subsection{The Transfer Function}

$H(\omega)$ represents is a particular kind of what is known mathematically as a transfer function, the Frequency Response Function (FRF), commonly used in Structural Engineering. the exact analytical definition of an FRF depends on the measured signal (force, displacement, velocity or acceleration) utilised in input and output. For illustrative purposes, let the input be a force $F(\omega)$ and the output a time history of displacements, such that $H(\omega)$ express the frequency-wise amount of displacement per unit of force applied. This case is generally referred to as the receptance FRF [3], the measurement unit of which is the displacement $(\mathrm{m})$ over force $(\mathrm{N})$. Without loss of generalisation, let also be the system under scrutiny defined as a single-degree-of-freedom (SDoF), the equation of motion of which can be simply stated as

$$
m \ddot{x}(t)+c \dot{x}(t)+k x(t)=f(t)
$$

where $m, c, k$ are, in the same order, the mass, viscous damping, and stiffness of the system. $x(t)$ is the time-dependent displacement, while its first and second timederivatives, $\dot{x}(t)$ and $\ddot{x}(t)$, are respectively the velocity and the acceleration of the same structural response. Finally, $f(t)$ stands for the external driving force.

One way of deriving the dynamic response of a system under different types of excitation is utilising the Laplace transform method. The Laplace transform of a function $x(t)$, denoted as $X(s)$ can be written as

$$
X(s)=\mathscr{L}[x(t)]=\int_{0}^{+\infty} e^{-s t} x(t) d t
$$


where $s$ is a complex parameter called the Laplace variable. Applying the Laplace transform defined in Eq (3) to the left side of the equation of motion expressed in Eq (2) leads to

$$
\begin{gathered}
\mathscr{L}[m \ddot{x}(t)+c \dot{x}(t)+k x(t)]=m\left[s^{2} X(s)-s x(0)-\dot{x}(0)\right]+c[s X(s)-x(0)]+k X(s)= \\
\left(m s^{2}+c s+k\right) X(s)-m s x(0)-m \dot{x}(0)-c x(0)
\end{gathered}
$$

and considering for the right side that

brings to

$$
\mathscr{L}[f(t)]=F(s)
$$

$$
\left(m s^{2}+c s+k\right) X(s)=F(s)+m \dot{x}(0)+(m s+c) x(0)
$$

where $x(0)$ and $\dot{x}(0)$ are initial displacement and velocity respectively and the right side of Eq (5) can be regarded as a generalised transformed excitation. If the initial conditions are zero, which is equivalent to ignoring the solution of the homogeneous equation, the ratio of the transformed response to the transformed input force can be defined as a transfer function with the real part of the Laplace variable nullified, i.e. [13]

$$
H(s)=\frac{X(s)}{F(s)}=\frac{1}{m s^{2}+c s+k}
$$

which is the analytical definition of the system transfer function for the specific case of the receptance of an SDOF system. $H(s)$ is a complex function of $\mathrm{s}$ and represented as a surface in the Laplace domain. The values $s_{1}$ and $s_{2}$ of the characteristic equation can be stated as

$$
s_{1,2}=\sigma \pm i \omega_{d}
$$

with

$$
\sigma=-\zeta \omega_{n}
$$

and

$$
\omega_{d}=\omega_{n} \sqrt{1-\zeta^{2}}
$$

Being $\zeta$ the damping ratio, $\omega_{n}=\sqrt[2]{\mathrm{k} / \mathrm{m}}$ the natural angular frequency of the system, and $\omega_{d}$ the damped counterpart of $\omega_{n}$. The transfer function can now be rewritten as

$$
H(s)=\frac{1}{m\left(s-s_{1}\right)\left(s-s_{2}\right)}
$$

where $s_{1}=\sigma+\mathrm{i} \omega_{\mathrm{d}}$ and $s_{2}=s_{1}^{*}=\sigma-i \omega_{d}$ are the so-called poles of the transfer function; thus, it can be further restated as

$$
H(s)=\frac{1}{m\left(s-s_{1}\right)\left(s-s_{2}\right)}=\frac{A}{\left(s-s_{1}\right)}+\frac{A^{*}}{\left(s-s_{1}^{*}\right)}
$$

where $A$ is given by

$$
A=-\frac{1}{i 2 m \omega_{d}}
$$

and together with its complex conjugate $A^{*}$ form the residues of the same. Importantly, residues and poles can be real quantities or come in complex-conjugate pairs. Before seeing how these two quantities are extracted by means of the VF approach, a couple of theoretical points still need to be addressed. 


\subsection{Several Definitions of the Transfer Function}

Several concurring alternatives exist to approximate the transfer function $H(s=$ $\left.\sigma \pm i \omega_{d}\right)$ directly in the Laplace domain or when transferred in the frequency domain. As they all serve the same aim and represent the same physical meaning, all these variants are interchangeable; yet they have unique properties which do not necessarily translate from one writing to another.

Regardless of its specific ambit of application, any transfer function expressed in terms of $s$ or $\omega$ can be considered as a rational polynomial, an ensemble of partial fractions, or a pole-zero system, that is to say

$$
\begin{aligned}
& H(s)=\frac{N(s)}{D(s)}=\frac{a_{0}+a_{1} s+a_{2} s^{2}+\cdots+a_{M} s^{M}}{b_{0}+b_{1} s+b_{2} s^{2}+\cdots+b_{N} s^{N}} \\
& H(s)=\sum_{n=1}^{N} \frac{c_{n}}{s-a_{n}}+H_{\infty} \\
& H(s)=H_{\infty} \frac{\left(s-z_{1}\right)\left(s-z_{2}\right) \ldots\left(s-z_{M}\right)}{\left(s-p_{1}\right)\left(s-p_{2}\right) \ldots\left(s-p_{N}\right)}
\end{aligned}
$$

$$
\begin{gathered}
\text { Rational Polynomial } \\
\text { Partial fractions } \\
\text { Pole-zero }
\end{gathered}
$$

The cases of major interest for this study are Eq (15), which is at the basis of the Vector Fitting method, and Eq (14), which is the definition of the commonly used Rational Fraction Polynomial (RFP) method [14]. In fact, the RFP is a methodology of proven and well-established efficacy in Civil and Mechanical Engineering and will be used in the next Sections as a mean of comparison. In the cases of Eq (14) and Eq (16), the orders of the numerator $N(s)$ and denominator $D(s)$ polynomials (respectively, $M$ and $N$ ) are independent of each other.

\subsection{The Vector Fitting Algorithm}

As the name itself suggests, the technique works by fitting a vector of transfer functions belonging to the same system. Therefore, the VF can be applied for single-input single-output (SISO) system identification; yet its natural implementation is for singleinput multi-output (SIMO) identification, which leads to a more robust solution. The aim is to construct a single rational function, expressed in terms of poles and residues, which fully represents the system investigated up to the arbitrarily set order. The result is what is called a surrogate model (also known as meta- or macro-model) of the system.

The original concept of fitting a rational model to frequency domain data can be traced back at least to the early 60s [15], until the works initiated by Gustavsen and Semlyen in the late 90s [4] which form the framework of this discussion.

A variant of the Fast Relaxed Vector Fitting (FRVF) procedure [5] has been here implemented, adapted to the needs of mechanical vibrations. Since neither the FRVF nor the original algorithm proposed in [4] has ever been applied to mechanical systems, the latter will be outlined before discussing the FRVF improvements.

\subsubsection{Basic VF Formulation}

To be coherent with the terminology utilised in [4] and following works, Eq (15) may be defined as

$$
f(s) \approx \sum_{n=1}^{N} \frac{c_{n}}{s-a_{n}}+d+s e
$$


where $a_{n}$ coincides with the $n$-th pole, $c_{n}$ with the corresponding residue, and $H_{\infty}$ is split into the two real quantities $d$ and $e$. It is important to remark how the modal parameters can be then extracted from these estimated poles and residues since each $n$-th pole contains information about the modal damping $\zeta_{n}$ and the natural frequency $f_{n}$ of its corresponding mode, while its residue is related to its mode shape (defined by the vectors $\varphi_{n}$ in the matrix $\left.\Phi=\left[\varphi_{1}, \varphi_{2}, \ldots \varphi_{N}\right]\right)$, i.e.

$$
f_{n}=\frac{1}{2 \pi} \sqrt[2]{\operatorname{Re}\left(a_{n}\right)^{2}+\operatorname{Im}\left(a_{n}\right)^{2}}, \zeta_{n}=\frac{-\operatorname{Re}\left(a_{n}\right)}{\sqrt[2]{\operatorname{Re}\left(a_{n}\right)^{2}+\operatorname{Im}\left(a_{n}\right)^{2}}}, \Phi=C \Psi
$$

Where $C$ is the vector of the residues and $\Psi$ is the matrix of the right eigenvectors of the state matrix of the system.

To begin with, it is needed to initialise a first attempt selection of the poles' location. These are therefore denominated initial poles. After that, the poles are relocated iteratively, and the corresponding residues are computed. The procedure is stopped when the root mean square error (RMSE) of the fitting goes behind an arbitrarily selected threshold, or when the iteration counter reaches a maximum number defined previously.

Regarding the selection of the initial poles, any set of pairs of complex conjugates with weak attenuation can be chosen. The weak attenuation is needed to ensure that the Least Square (LS) problem [16] has a well-conditioned system matrix. In detail, the imaginary part of the several complex pairs should cover the frequency range of interest, to reduce the probability of relocating the poles over a long distance; it is convenient to choose the initial poles $\tilde{a}_{n}$ with imaginary part $\beta$ linearly spaced throughout the frequency band of the observation, such as that

$$
\tilde{a}_{n}=-\alpha+\mathrm{j} \beta, \tilde{a}_{n+1}=-\alpha-\mathrm{j} \beta
$$

with $\alpha=\beta / 100$, to depart from initial poles with a small real part, reducing the risk of problem ill-conditioning [17]. The negative real part is needed to enforce the stability of the initial poles, as the eigenvalues of state matrix $A$ need to have a negative real part to be stable.

Once the series of starting poles $\tilde{a}_{n}$ is set, the fitting procedure continues as follow. Let $f(s)$ be the transfer function of interest, multiplied with an unknown function $\sigma(\mathrm{s})$ defined by the following approximation as:

$$
\sigma(s) \approx \sum_{n=1}^{N} \frac{\tilde{c}_{n}}{s-\tilde{a}_{n}}+1
$$

The following problem can be stated:

$$
\left[\begin{array}{c}
\sigma(s) f(s) \\
\sigma(s)
\end{array}\right] \approx\left[\begin{array}{c}
\sum_{n=1}^{N} \frac{c_{n}}{s-\tilde{a}_{n}}+d+s \cdot e \\
\sum_{n=1}^{N} \frac{\tilde{c}_{n}}{s-\tilde{a}_{n}}+1
\end{array}\right]
$$

The rational approximation of $\sigma(s)$ has the same poles of the approximation $\sigma(s) f(s)$. The ambiguity in the solution for $\sigma(s)$ is removed by forcing $\sigma(s)$ to approach unity at very high frequencies, i.e., by imposing $H_{\infty}=1$. Then, substituting Eq (20) in Eq (21) leads to 


$$
\sigma(s) f(s) \approx\left(\sum_{n=1}^{N} \frac{\tilde{c}_{n}}{s-\tilde{a}_{n}}+1\right) f(s) \approx \sum_{n=1}^{N} \frac{c_{n}}{s-\tilde{a}_{n}}+d+s \cdot e
$$

which is linear in its the unknown $c_{n}, d, e, \tilde{c}_{n}$. This represents the relation

$$
(\sigma f)_{f i t}(s) \approx(\sigma)_{f i t}(s) f(s)
$$

Eq (23) can be rewritten in turn as

$$
\left(\sum_{n=1}^{N} \frac{c_{n}}{s-\tilde{a}_{n}}+d+s \cdot e\right)-\left(\sum_{n=1}^{N} \frac{\tilde{c}_{n}}{s-\tilde{a}_{n}}\right) f(s) \approx f(s)
$$

Which is an explicit formulation for the rational function approximation of $f(s)$ which can be solved as a least-square problem. Indeed, this is clear if each sum of partial fractions in $\mathrm{Eq}(23)$ is written as a fraction:

And thus

$$
(\sigma f)_{f i t}(s)=e \frac{\prod_{n=1}^{N+1}\left(s-z_{n}\right)}{\prod_{n=1}^{N}\left(s-\tilde{a}_{n}\right)}, \quad \sigma_{f i t}(s)=\frac{\prod_{n=1}^{N}\left(s-\tilde{z}_{n}\right)}{\prod_{n=1}^{N}\left(s-\tilde{a}_{n}\right)}
$$

$$
f(s)=\frac{(\sigma f)_{f i t}(s)}{\sigma_{f i t}(s)}=\frac{\prod_{n=1}^{N+1}\left(s-z_{n}\right)}{\prod_{n=1}^{N}\left(s-\tilde{a}_{n}\right)} \cdot e \frac{\prod_{n=1}^{N}\left(s-\tilde{a}_{n}\right)}{\prod_{n=1}^{N}\left(s-\tilde{z}_{n}\right)}=e \frac{\prod_{n=1}^{N+1}\left(s-z_{n}\right)}{\prod_{n=1}^{N}\left(s-\tilde{z}_{n}\right)}
$$

As $\tilde{a}_{n}$ cancel out in the division, being them the initial poles of both $(\sigma f)_{f i t}$ and $\sigma_{f i t}(s)$. Importantly, this proves that the poles of $f(s)$ become equal to the zeros of $\sigma_{f i t}(s)$.

An important outcome derives from this formulation. In the case of transfer functions, $f(s)=H(s)$ can be described as a ratio between two functions (the input as the denominator and the output as the numerator), both of which can, in turn, be approximated as partial functions, that is to say

$$
H(s)=\frac{N(s)}{D(s)} \approx \frac{\prod_{n}\left(s-z_{n}\right)}{\prod_{n}\left(s-\tilde{z}_{n}\right)}=\frac{\frac{\prod_{n}\left(s-z_{n}\right)}{\prod_{n}\left(s-a_{n}\right)}}{\frac{\prod_{n}\left(s-\tilde{z}_{n}\right)}{\prod_{n}\left(s-a_{n}\right)}}=\frac{d+\sum_{n=1}^{N} \frac{c_{n}}{s-a_{n}}}{d^{\prime}+\sum_{n=1}^{N} \frac{c_{n}^{\prime}}{s-a_{n}}}
$$

The relation between the true transfer function, $H$, and the fitted residues, $\left[c_{n}, c^{\prime}{ }_{n}\right]$, poles $\left[a_{n}, a_{n}^{\prime}\right]$, and constant terms $\left[d, d^{\prime}\right]$ can be then written as:

$$
d+\sum_{n} \frac{c_{n}}{s-a_{n}} \approx H(s)\left(d^{\prime}+\sum_{n} \frac{c^{\prime}{ }_{n}}{s-a_{n}}\right)
$$

Therefore, the fitting problem, which is nonlinear if approximated as rational polynomials (Eq (14)), becomes a (weighted) linearised minimisation [18]; this also avoids the severe ill-conditioning which derives from the rational polynomial approximation, where it can be easily proved that the approximated $H(s)$ turns out to be a Vandermonde matrix with all the inherent problematics.

In summary, the pole-relocation process is a linear solution that is made possible by assuming a basis consisting of partial fractions. This is evaluated by dividing the numerator and denominator of the rational polynomial approximation by a common set of poles. That does not change the nature of the problem but allows to reformulate it such that all unknowns are linear. The (linear) minimisation task can be therefore solved iteratively. 


\subsubsection{Fast Relaxed VF}

The twofold intent here is (i) to add a relaxed non-triviality constraint in the pole identification stage of Vector Fitting for acquiring faster convergence and less biasing [9], also improving its convergence performance especially in presence of noise, while (ii) solving the linear problem related to the pole identification stage of Vector Fitting accordingly to the $\mathrm{QR}$ decomposition approach. The $\mathrm{QR}$ decomposition [19,20] exploits the sparsity of the least-square equations and is a technique increasingly appreciated in the ambit of the structural identification and model reduction. This approach performs much faster than the original VF fitting technique.

By recalling Eq (20), it is possible to remove the high-frequency asymptotic constraint on $\sigma(s)$, replacing the unit value with a real-valued free variable $\hat{d}$, i.e.

$$
\sigma(s)=\sum_{n=1}^{N} \frac{\tilde{c}_{n}}{s-a_{n}}+\hat{d}
$$

which from here on will be referred to as the Relaxed formulation of Vector Fitting (RVF) [9]. It is then possible to replace Eq (20) with Eq (29) and to follow the derivation steps presented in the previous subsection (steps omitted for brevity's sake). However, to avoid a null (trivial) solution, it is necessary to add one equation to the LS problem:

$$
\operatorname{Re}\left\{\sum_{k=1}^{N_{s}}\left(\sum_{n=1}^{N} \frac{\tilde{c}_{n}}{s_{k}-a_{n}}+\hat{d}\right)\right\}=N_{s}
$$

Eq (30) enforces that the sum of the real part of $\sigma(s)$ over the given $N_{s}$ frequency samples equal some nonzero values, without explicitly defining any of the free variables. Eq (30) should also be weighted as

$$
\text { weight }=\frac{\|w(s) \cdot f(s)\|_{2}}{N_{s}}
$$

where $w(s)$ is an arbitrarily chosen set of weights.

Two major advantages derive from replacing the constraint of the original formulation of $\mathrm{VF}$ with this milder summation requirement:

1. The arbitrary choice of the initial poles is (much) less critical, especially in case of noisy measurements [9];

2. The convergence of the problem is significantly increased [8].

Both these points are achieved by letting the scaling function $\sigma(s)$ free to attain any value. In fact, if the set of initial poles is poorly defined, large pole shifts may be needed in the first iterations. This results in the magnitude of $\sigma(s)$ becoming much larger than unity in some frequency bands. Noteworthy, as RVF converges throughout the iterations, $\sigma(s)$ will nevertheless approach unity at all frequencies and the residues will be zeroed $\left(\left\{\tilde{c}_{n}\right\}=0, \hat{d}=1\right)$ similarly as in the original VF formulation of Eq (20). It follows that the additional criterion $\mathrm{Eq}$ (30) does not impose any constraint on the LS problem other than preventing it from becoming null. On the other hand, the RVF formulation reduces the magnitude of pole relocation bias by allowing the constant term of $\sigma$ to take on any real value to compensate for large residue coefficients. 
Since Eq (29) does not approach unity at high frequencies, the eigenvalue problem which defines the poles at any iteration step should be slightly reformulated as

$$
\left\{a_{n}\right\}=\operatorname{eig}\left(A-b \cdot \hat{d}^{-1} \cdot c^{T}\right)
$$

where for $N$ states, $A=\operatorname{diag}\left(a_{n}\right)$ is the $N$-by- $N$ state matrix of the system, $b$ is a column vector of $1 \mathrm{~s}$ such that $A \mathrm{X}=b$, and $c^{T}$ is a row vector containing the residues. The zeros calculation by Eq (32) is only applicable with a non-zero $\hat{d}$. If the absolute value of $\hat{d}$ is found to be smaller than a given tolerance tol, the solution is discarded and the LS problem is solved again with a fixed value for $\hat{d}$ as

$$
\hat{d}=t o l \cdot(\hat{d} / a b s(\hat{d}))(33)
$$

The second improvement with respect to the basic VF algorithm consists of an application of the QR factorisation to the single-element LS equations. This step leads to a simplified set of equations; in turn, these equations are computed for each matrix element to identify a common pole set. Basically, the QR factorisation operates by decomposing the matrix of LS equations $L S$ into the product $L S=Q \cdot R$. In fact, the overdetermined set of equations is very sparsely populated, since it is defined by

$$
\left[\begin{array}{ccccc}
X & 0 & 0 & \ldots & -H_{1} X \\
0 & X & 0 & \ldots & -H_{2} X \\
\vdots & \vdots & \vdots & \ddots & \vdots \\
0 & 0 & 0 & X & -H_{V} X
\end{array}\right]\left[\begin{array}{c}
C_{1} \\
C_{2} \\
\vdots \\
C_{V} \\
\tilde{C}
\end{array}\right]=\left[\begin{array}{c}
H_{1} \hat{1}_{K} \\
H_{2} \hat{1}_{K} \\
\vdots \\
H_{V} \hat{1}_{K}
\end{array}\right]
$$

Where $H_{v}$ is derived from the $v$-th the set of measured responses, $C_{v}$ is the $v$-th vector that contains the residues of $(\sigma f)_{v}, \tilde{C}$ contains the residues $\sigma(s), \hat{1}_{K} \in \mathbb{R}^{K}$ indicates a column vector of ones with the same length as the number of frequency samples included, and

$$
X=\left[\begin{array}{ccccc}
\frac{1}{s_{1}-a_{1}} & \frac{1}{s_{1}-a_{2}} & \cdots & \frac{1}{s_{1}-a_{N}} \\
\frac{1}{s_{2}-a_{1}} & \frac{1}{s_{2}-a_{2}} & \cdots & \frac{1}{s_{2}-a_{N}} \\
\vdots & \vdots & & \ddots & \vdots \\
\frac{1}{s_{K}-a_{1}} & \frac{1}{s_{K}-a_{2}} & \cdots & \frac{1}{s_{K}-a_{N}}
\end{array}\right]
$$

for a rational function of order $N$ and $K$ frequency samples. The columns of the matrix $Q$ form an orthonormal basis that span the column space of $L S$ through the coefficients embedded in the (right) triangular matrix $R$. Analytically, this coincides with the poleidentification of each matrix element $v=1, \ldots, V[5]$ :

$$
\left[\mathrm{X}-H_{v} \mathrm{X}\right]=\left[Q_{v}\right]\left[\begin{array}{cc}
R_{v}^{11} & R_{v}^{12} \\
0 & R_{v}^{22}
\end{array}\right]
$$

combining the factorization for all matrix a reduced set of equations is obtained:

$$
\left[\begin{array}{c}
R_{2}^{22} \\
\ldots \\
R_{V}^{22}
\end{array}\right][\tilde{C}]=\left[\begin{array}{c}
Q_{1}^{T} H_{1} \hat{1} \\
\ldots \\
Q_{V}^{T} H_{V} \hat{1}
\end{array}\right]
$$

where the shared coefficients $\tilde{C}$ of $\sigma(s)$ are the only unknowns of the reduced system in Eq (37) and the LS matrices on the left half side of both Eq (36) and Eq (37) are smaller than the sparse matrix definition which results from the overdetermined set of equations 
in Eq (34). Exploiting the sparsity of the LS matrices in such way leads to a significant reduction in terms of computation time, flops requirements, and memory storage. Importantly, the gain of resorting to this compressed formulation increases with the overdetermination of the problem, i.e. for an increasing number of samples per response and/or the total number of output channels. This makes the whole process more convenient for robust system identification with many output channels. Due to space limitations, it is not possible to better detail the QR factorisation and its interesting properties; the interested reader can find a much deeper discussion in [5] (Section III) and [21] (which also reports a very reader-friendly graphical explication of the procedure).

\subsubsection{Implementation Procedure}

To summarise, the proposed application of the FRVF algorithm in the context of a mechanical system can be defined as a five steps input-output procedure:

1. Apply and measure the input(s) at one or more source location(s);

2. Collect the measured output(s) at one or more channel(s);

3. Compute the FRFs with the unit of measure of interest (displacement, velocity, acceleration, or force, both in input and in output), converting the acquired signals if necessary;

4. Apply the FRVF scheme summarised by the following substeps:

a. Select the initial poles $\tilde{a}_{n}, n=1, \ldots, N$;

b. Solve the linear relaxed LS problem via QR decomposition, utilising the poles of the previous iterations;

c. Find the zeros of the denominator $D(s)$ as a basic eigenvalue problem;

d. Set the new poles $a_{n}$;

e. Solve by iterating b-d until convergence.

5. Extract the modal parameters (natural frequency, damping ratio, and mode shape) from each identified mode (i.e. from each conjugated pair of poles).

Please notice that omitting the relaxing condition and QR decomposition in 4.b, the same procedure can be applied for the standard VF algorithm.

\subsubsection{Comparison with Frequency-Domain and Time-Domain SI Techniques}

To prove and establish the capabilities of the FRVF method, a comparison with the RFP will be used in Section 5, as mentioned earlier.

For further comparison, the Stochastic Subspace Identification (SSI) [22], was applied. Specifically, the MatLab® N4SID function was utilised for input-output timedomain SI. This is a necessary act, as SSI-derived techniques are, arguably, the standard for linear SI in common practice and they are widely considered as the default choice for mechanical systems. Yet, the use of SSI-based approaches is hampered in other sectors, such as for the identification of electrical circuits, by the fact that they become computationally too expensive for a large number of input and output channels. Without going too much into details (a deeper discussion can be found in [23]), this methodology considers the state-space formulation associated with the equation of motion, i.e. 


$$
u_{k+1}=A u_{k}+e_{k} \quad k=0,1,2, \ldots
$$

And builds large-block Hankel matrices from the output sequence, divided into past and future data matrices. Then, by projecting the row space of the future block Hankel matrix into the row space of the past block Hankel matrix, the corresponding Kalman filter state sequence can be obtained, and the modal parameters of the structure can be estimated by solving a least-square optimisation [24].

\section{Numerical Case Studies.}

A 9 degrees-of-freedom (DoFs) shear-type frame structure has been simulated numerically for the preliminary validation of the proposed procedure. The structure is defined by lumped, story-wise mass $m_{i}=1 \mathrm{~kg}$ and $i$-th inter-story stiffness $k_{i}=$ $5000 \mathrm{~N} / \mathrm{m}$. A sampling frequency $f_{s, \text { num }}=200 \mathrm{~Hz}$ and a frequency resolution of $\Delta f_{\text {num }}=0.05 \mathrm{~Hz}$ were simulated. A unit $(1 \mathrm{~N})$ rectangular impulse has been applied to the first floor as input force. The receptance FRFs were calculated considering the ratio of the FFT of the displacement output at any story over the FFT of the external force, as defined in Eq (7). The aim of this numerical investigation was (i) to investigate the effects of noise and (ii) to discern modelling errors (biases) from measurement errors. The numerical case also allowed to study a (simulated) system with a well-known and finite number of modes.

\subsection{Numerical Investigation of Noise Effects}

The convergence properties of FRVF on numerical data, in particular when the frequency samples are affected by noise (artificially added in the input and the output's signals), have been analysed. It is known that even small amounts of noise are disruptive and seriously impair or destroy convergence. This is due to the presence of spurious poles that appear during the iterations and might try to fit the noise instead of the real data [25]. The "pseudo-experimental" signals, intended to mimic the measurement error of actually recorded data, have been defined (in the time domain) by adding to the input and/or output a noise equal to a percentage of its (stationary) standard deviation. This percentage has been raised from $0 \%$ to $20 \%$. It must be reminded that a percentage of noise equal to $2 \%$ corresponds to a Signal-To-Noise-Ratio (SNR) value higher than $50 \mathrm{~dB}$, which corresponds to a very noisy signal in practical terms.

Results for the noise-free configuration, 1\%, 5\%, and 10\% are depicted in Figure 1 as an example. The (weighted) Root Mean Square Error (RMSE) was used as a measure for the deviation between the known ground truth and the model prediction, accordingly to the classic formulation

$$
R M S E=\sqrt[2]{\frac{1}{K} \sum_{k=1}^{K}\left|\left(f_{f i t}(k)-f(k)\right)\right|^{2}}
$$

with $f_{f i t}(k)$ as the predicted value at frequency $k$ and $f(k)$ as the actual value for all the $\mathrm{K}$ frequency samples contained into each FRF.

In the following figures, the rational approximations $\sigma_{f i t}(\mathrm{~s})$ and $(\sigma f)_{f i t}(s)$ are reported, respectively, as solid blue and dashed red lines. The deviation between the 
magnitude of $(\sigma)_{f i t}(s) f(s)$ and $(\sigma f)_{f i t}(s)$ is illustrated in light green. The 9 output channels are all superimposed for compactness of representation. Figure 2 reports the pole location accordingly to these levels of noise; as expected, spurious poles appear more numerous as the noise increases.
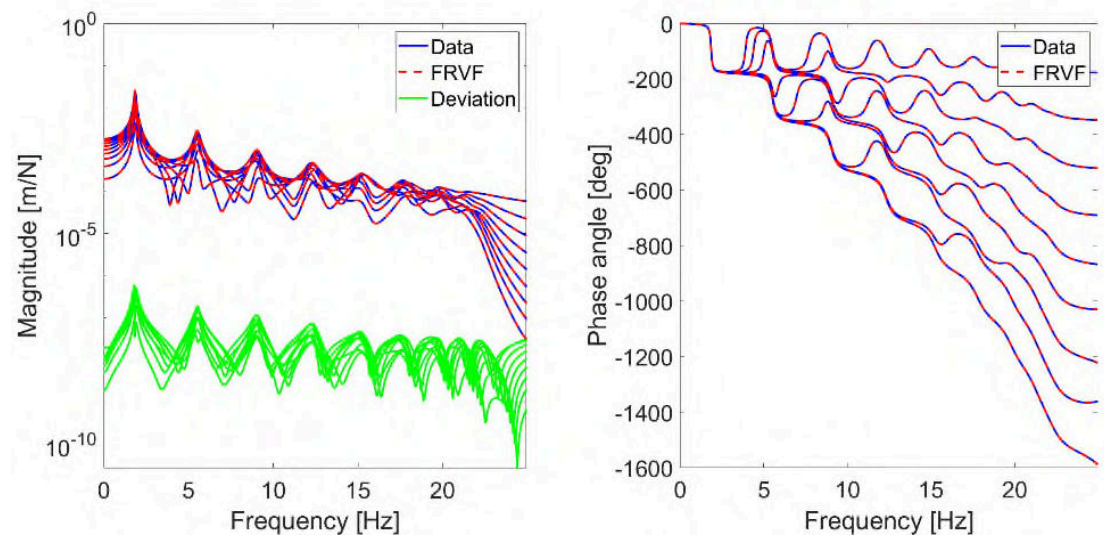

(a)
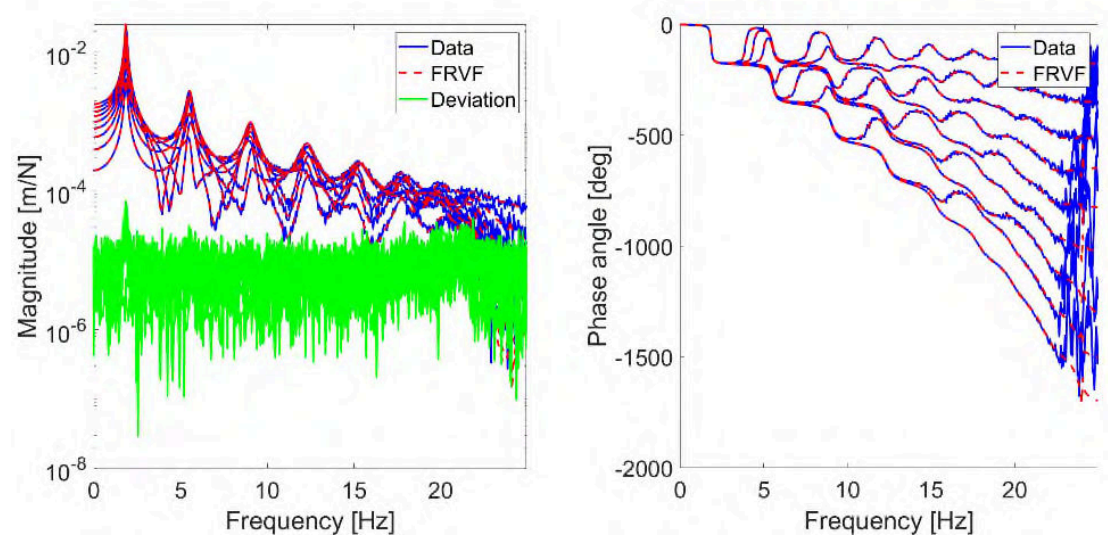

(b)
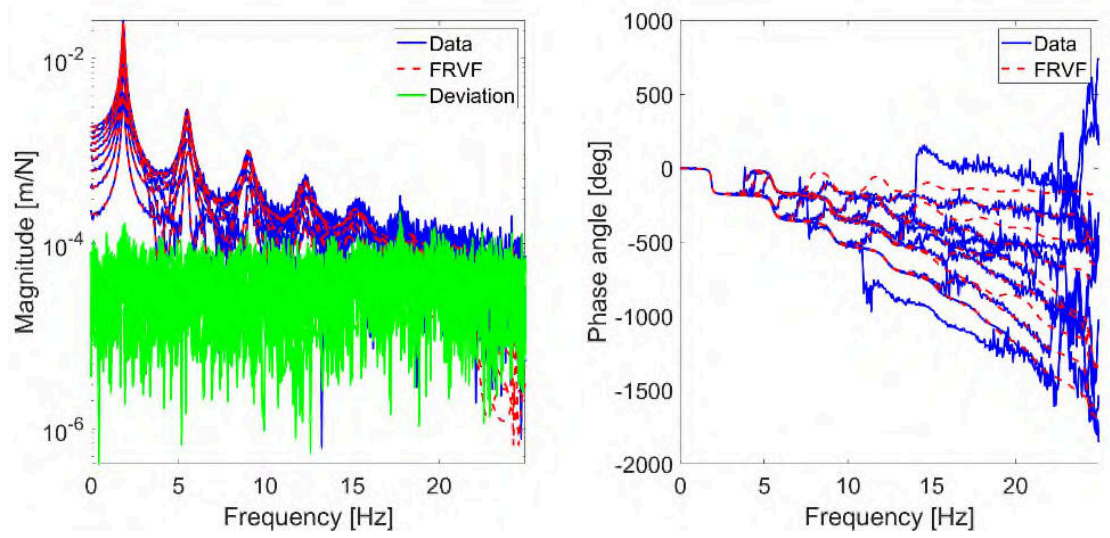

(c) 

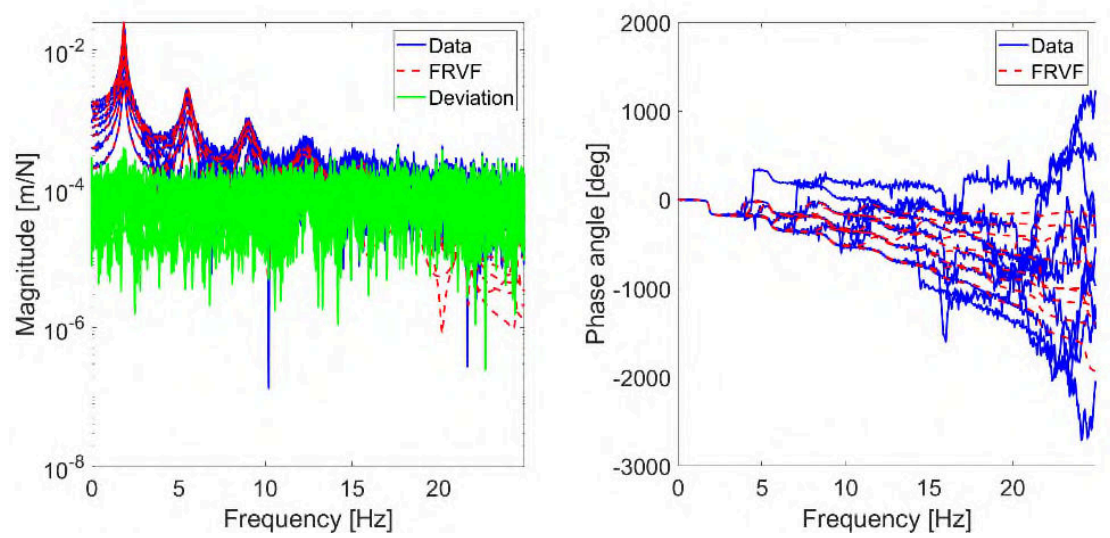

(d)
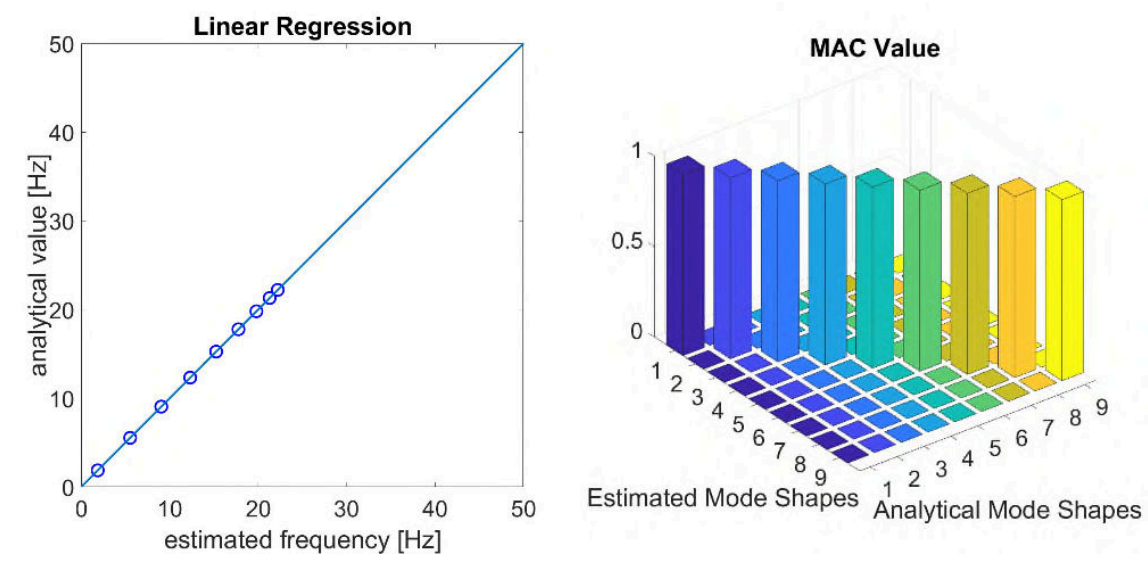

(e)

Figure 1. Numerical study: Fitting over the SIMO system with nine output channels (one per story). (a) Noise-free (b) $1 \%$ noise (c) $5 \%$ (d) $10 \%$. The plot of the absolute value of gain (left) and phase angle (right). (e) linear regression of the identified natural frequencies (left) and Modal Assurance Criterion (MAC) (right); case without noise.

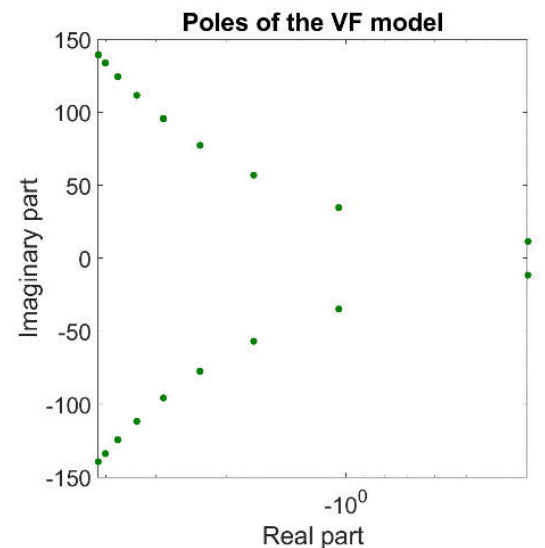

(a)

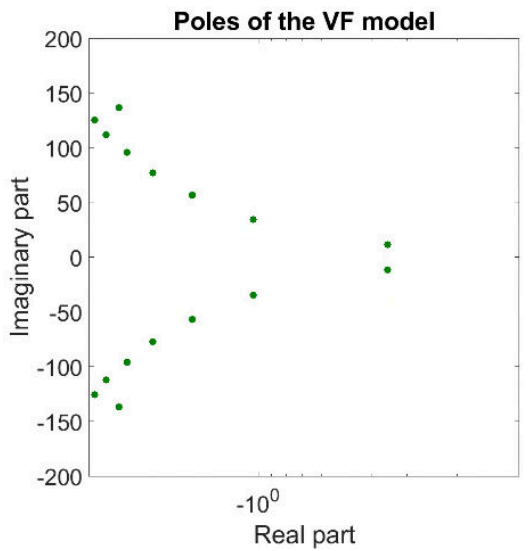

(b) 


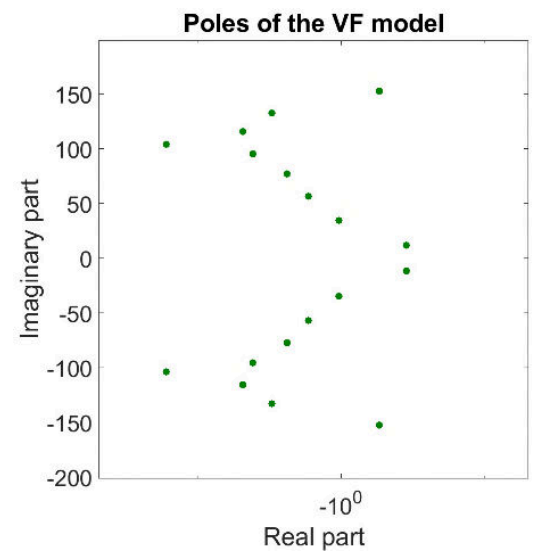

(c)

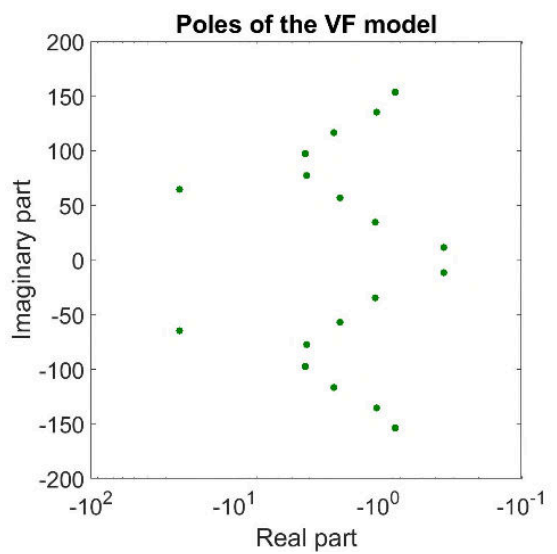

(d)

Figure 2. Numerical study: Identified poles of the SIMO system. (a) Noise-free (b) $1 \%$ (c) $5 \%$ (d) $10 \%$.

The overall results this numerical study on the simulated SIMO system are reported in Figure 3, respectively for noise in the input signals, in the output signals, and both. In all cases, the percentage error between FRVF results and known ground truth (computed numerically) is reported for natural frequencies, modal damping ratios, and the Modal Assurance Criterion (MAC, [26]) value of the mode shapes, defined as

$$
\operatorname{MAC}\left(\varphi_{A}, \varphi_{P}\right)=\frac{\left(\varphi_{A} \cdot \varphi_{P}\right)^{2}}{\left(\varphi_{A} \cdot \varphi_{A}\right)\left(\varphi_{P} \cdot \varphi_{P}\right)}(40)
$$

between the actual and the predicted mode shape (respectively, $\varphi_{A}$ and $\varphi_{P}$ ).

It is evident how, when only the input signal is corrupted by noise, the estimation error of the resonance frequencies is low: it is approximately equal to 1-5\% in the worst case (i.e. for the higher modes). The error on the damping increases similarly reaching a $40 \%$ error for the $8^{\text {th }}$ and $9^{\text {th }}$ mode. Accordingly to the MAC value, only the identification of the $9^{\text {th }}$ mode suffers significantly in this case.

For noisy output signals, the identification procedure becomes much more compelling. The error in terms of frequency estimation is approximately equal to $1-5 \%$ for a noise range of $1-10 \%$ but is higher than $20 \%$ for higher noise levels. The error in modal damping estimation affects a larger number of modes increasing the noise level and it is particularly noticeable for the higher modes; the same applies to the mode shapes. Finally, in the presence of noise in both the output and input signals, the FRVF procedure is still able to identify properly at least the parameters of the first two modes even in the harshest conditions. Also, for all modes, the absolute error is not too much greater than for the two previous scenarios.

Figure 4 points out how the RMSE at each iteration (always considering 5 iterations) increase linearly with the noise level for noise in input and output only, while it increases suddenly then remains almost stable in case of noise only in the input. Also, it is possible to notice how the iteration procedure converge always in maximum 3 iterations; the reached RMSE never exceeds $10^{-3}$, which can be considered accordingly to common practice and scholarly consensus a perfect fit. 

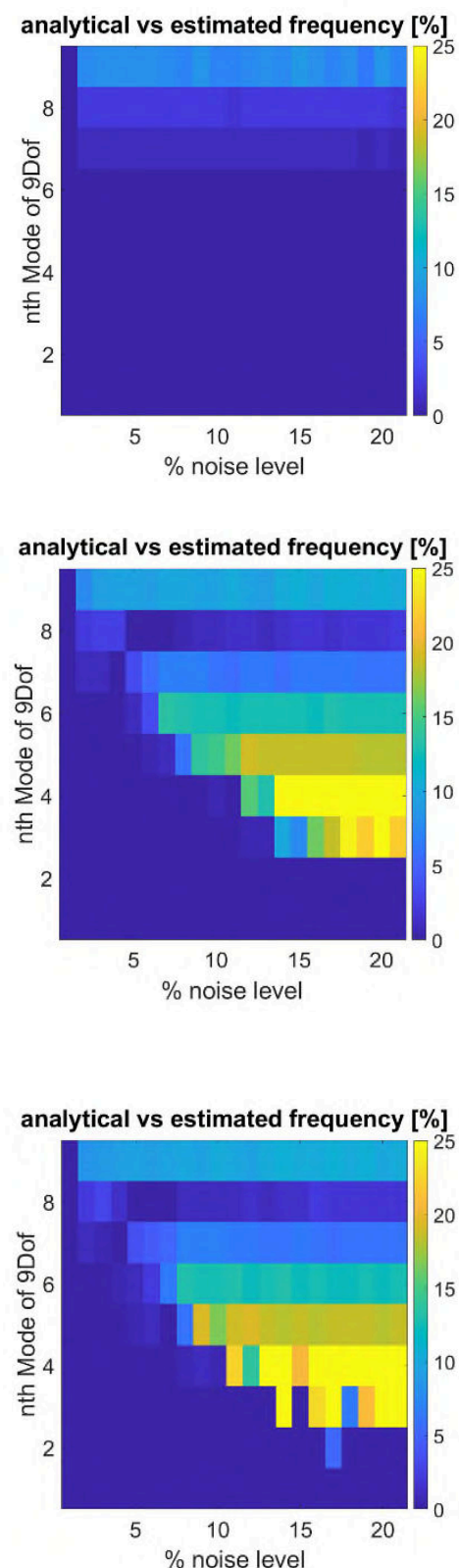

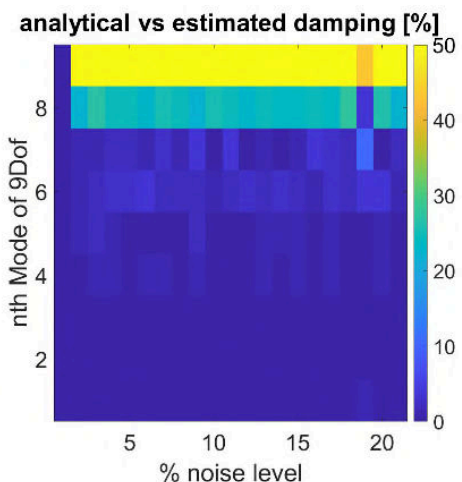

(a)

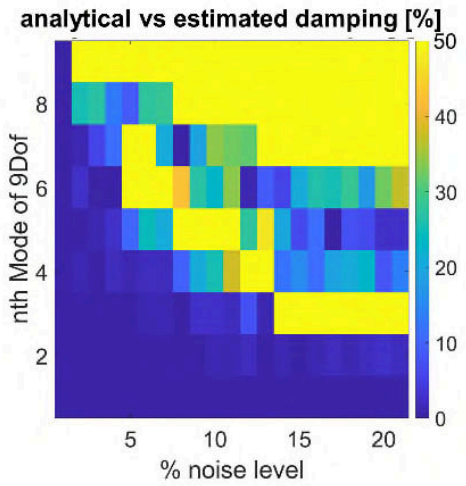

(b)

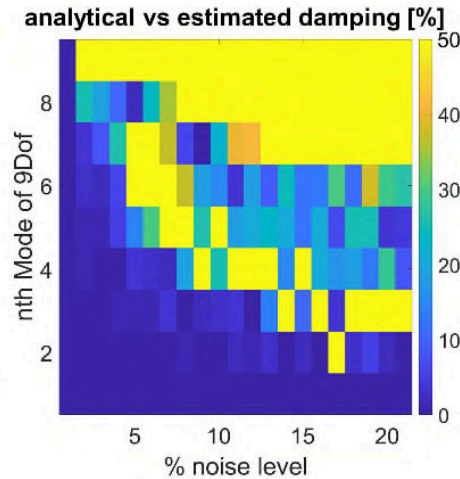

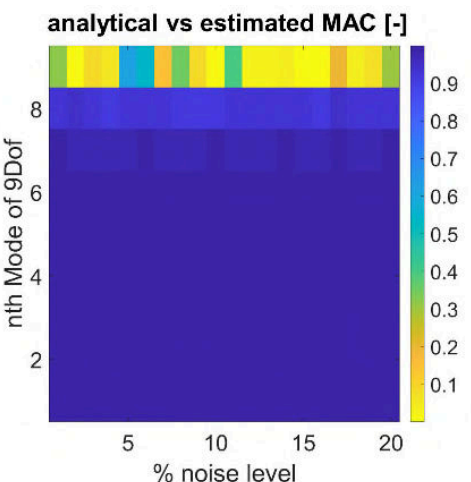
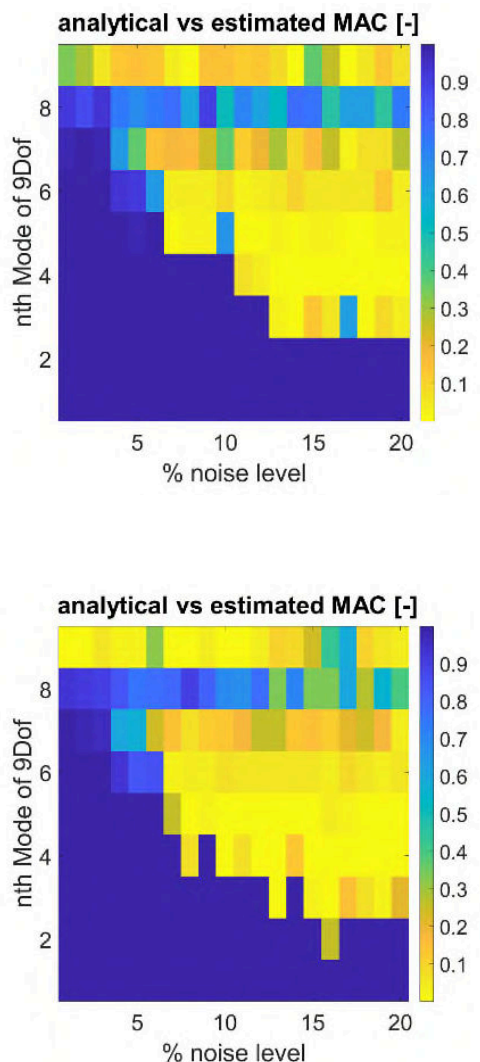

(c)

Figure 3. Numerical study: Error for increasing noise, artificially added in (a) the input signal, (b) the output signals, (c) in both the input and output signals. Left column: percentage error in natural frequency estimation. Middle column: percentage error in modal damping ratio. Right column: MAC value between numerical mode shapes and FRVF results.
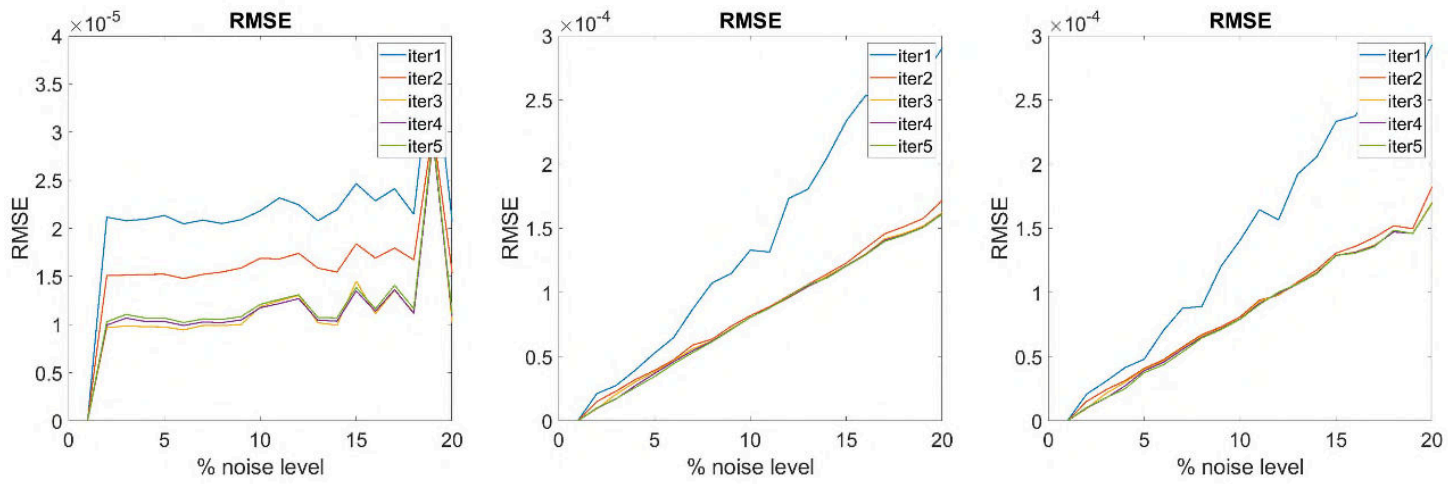
Figure 4. Numerical study: RMSE value to the increase of noise level and the number of iterations for each case studies: noise in input signals (left), noise in output signals (middle), noise in input and output (right)

\section{Preliminary Experimental Case Study.}

The experimental case study considered for the preliminary validation of the FRVF technique contemplates a HAR highly flexible wing prototype. Noteworthy, all tests were performed on the spar alone rather than on the actual skin-spar ensemble, as the innovative external skin - modelled in the framework of the BEARD project [27] - is supposed to transfer all the aerodynamical loads to the spar itself. Major details can be found in the inherent works $[28,29]$. The structure was considered when loaded with four inertial measurement units (IMUs) as depicted in Figure 5. The mechanical properties and the geometrical details for both the unloaded and sensor-loaded configurations are enlisted in Table 1. The acquisitions of acceleration time histories were performed at the four IMUs locations with a sampling rate of $f_{s, \exp 1}=100 \mathrm{~Hz}$. Therefore, due to the Nyquist criterion, only the first two bending modes were observable; higher modes fell out of range. The input was applied by a modal shaker at the clamped base as a $0.0015 \mathrm{~g}^{2} / \mathrm{Hz}$ white Gaussian noise defined between 2-60 Hz.

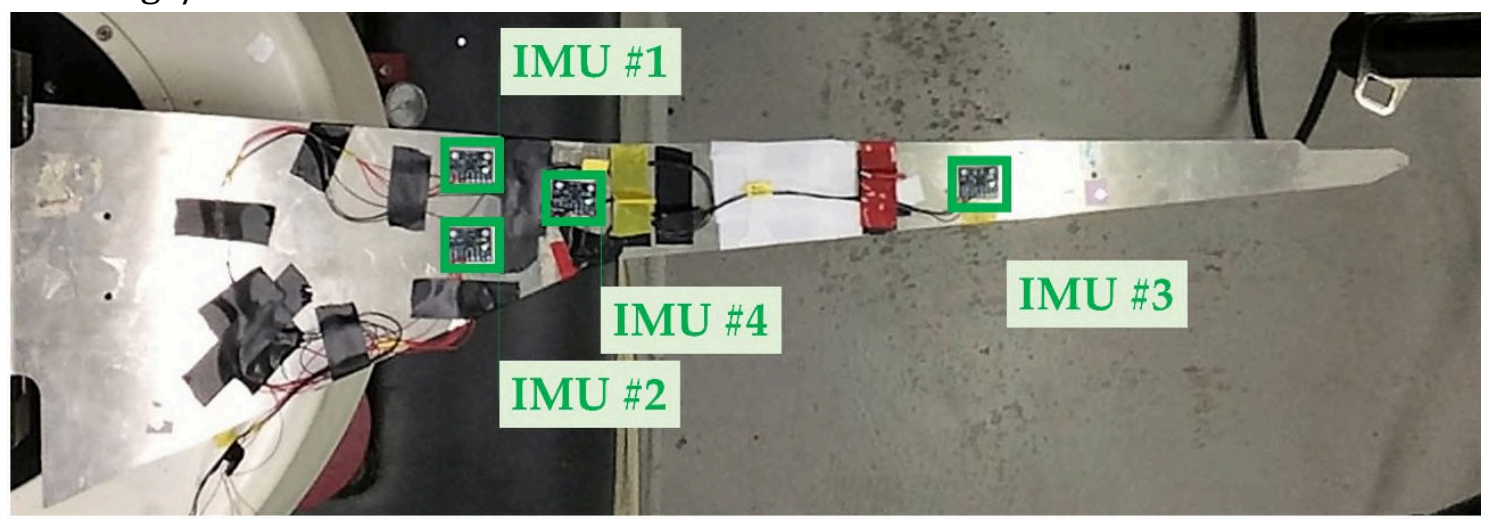

Figure 5. The HAR wing prototype spar with sensors attached.

\begin{tabular}{|c|c|c|}
\hline \multicolumn{3}{|l|}{ Geometrical Properties } \\
\hline Free length (clamp to tip) $l_{\text {tip }}$ & 706 & $\mathrm{~mm}$ \\
\hline Thickness $t$ & 2 & $\mathrm{~mm}$ \\
\hline Max width at clamped section $b_{\max }$ & 180.00 & $\mathrm{~mm}$ \\
\hline $\begin{array}{l}\text { Mid-length width at the section of changing tampering }(l=258 \\
\mathrm{mm}) b_{l=258}\end{array}$ & 56.10 & $\mathrm{~mm}$ \\
\hline Min width at the tip section $b_{\text {min }}$ & 17.04 & $\mathrm{~mm}$ \\
\hline \multicolumn{3}{|l|}{ Mechanical properties } \\
\hline Density $\rho$ & 2850 & $\mathrm{~kg} / \mathrm{m}^{3}$ \\
\hline Young's Modulus $E$ & $7.31 \cdot 10^{10}$ & $\mathrm{~Pa}$ \\
\hline Poisson Ratio $v$ & 0.33 & - \\
\hline
\end{tabular}

Table 1. Geometrical and mechanical properties of the wing spar. 


\subsection{Preliminary Experimental Results}

The modal identification of the wing spar produced results coherent with the calibrated FE model developed in [30] and applied in [31]. The investigation was bounded between 0 and $30 \mathrm{~Hz}$, such that just the first and the second modes could be detected; this was mainly due to the low sampling frequency of the attached IMU sensors. The modal parameters (natural frequency, modal damping, and mode shape normalised to the maximum displacement) are enlisted in Table 2, while the experimental mode shapes are portrayed in Figure 6 and benchmarked versus the calibrated FE model. To compare the results, the identified mode shapes have been normalised with respect to the displacement of the FE model at IMU\#3 for the $1^{\text {st }}$ mode shape and at IMU\#4 for the $2^{\text {nd }}$ mode shape.

The actual error in frequency estimation was around 5\%, with a MAC value of the extracted mode shapes of circa $95 \%$. These results were considered satisfactory in consideration of the relatively low-quality acquisitions of the low-cost, lightweight RaspBerry PI ${ }^{\circledR}$ Inertial Measurement Units (IMUs), which only had a sensitivity of 2048 LSB/g (least significant bit per g) [29]. The difference with the previously published results reported in [29] derives from the different input applied (white Gaussian noise rather than frequency sweeping).

\begin{tabular}{|c|c|c|c|c|c|}
\hline $\begin{array}{c}\text { Mode } \\
\text { number }\end{array}$ & $\begin{array}{c}\text { Frequency } \\
{[\mathrm{Hz}]}\end{array}$ & $\begin{array}{c}\text { Damping } \\
{[-]}\end{array}$ & \multicolumn{3}{|c|}{$\begin{array}{c}\text { Mode shape } \\
{[-]}\end{array}$} \\
\hline $\mathbf{1}$ & 5.00 & 0.0017 & $\begin{array}{c}-0.2241 \text { (IMU \#1), } \\
-0.2243 \text { (IMU \#2) }\end{array}$ & -0.3329 & -1 \\
\hline $\mathbf{2}$ & 21.36 & 0.0135 & $\begin{array}{c}0.8374(\text { IMU \#1), } \\
0.8202 \text { (IMU \#2) }\end{array}$ & 1 & 0.5923 \\
\hline
\end{tabular}

Table 2. HAR wing spar: modal parameters as identified by the FRVF technique.
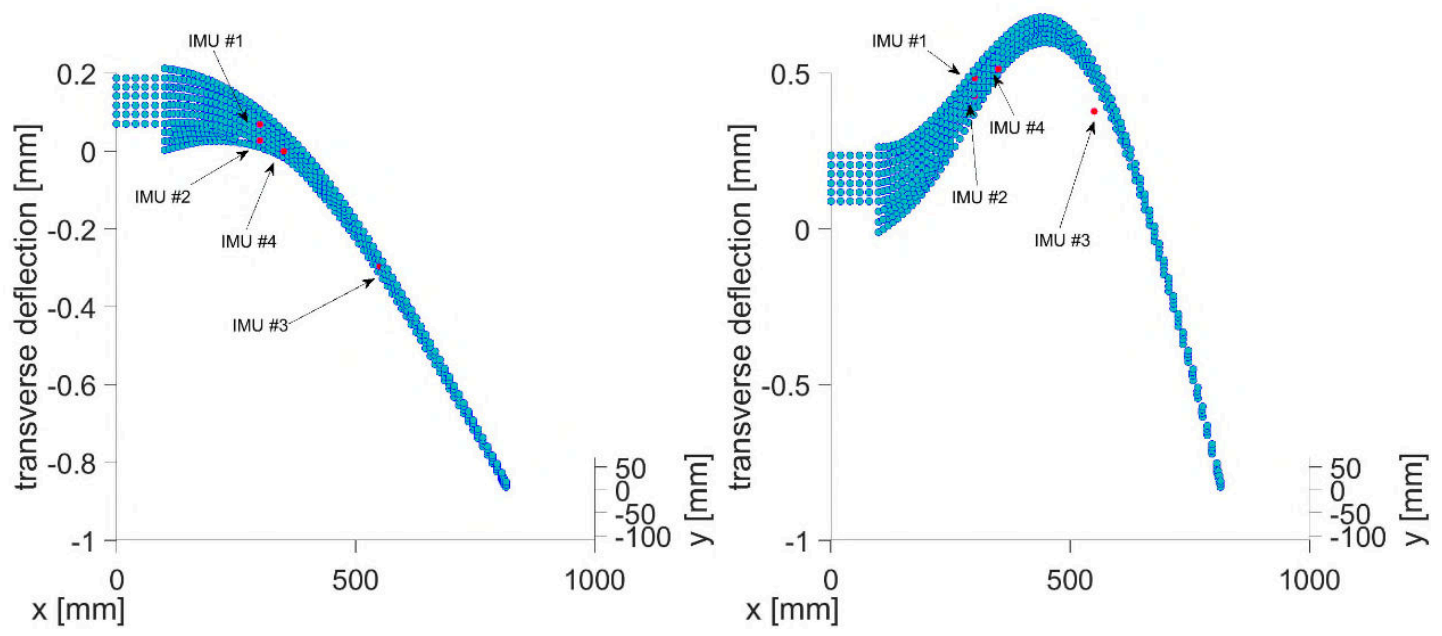

Figure 6. HAR wing spar. Left: first mode shape; right: second mode shape. The red dots indicate the results from Table 1 scaled appropriately; the blue dots represent the calibrated model described in [30]. 


\section{Main Experimental Case Study.}

The main Case Study is the well-known benchmark experiment performed at the Engineering Institute (EI) at the Los Alamos National Laboratory (LANL) [32]. The three-storey frame is characterised by aluminium columns and plates assembled through bolted joints, which slides on rails permitting movement in the x-direction only (see Figure 7). At each floor, four aluminium columns (with dimensions of $17.7 \times 2.5 \times 0.6$ $\mathrm{cm})$ are connected to the bottom and top aluminium plates $(30.5 \times 30.5 \times 2.5 \mathrm{~cm})$ forming a four DoFs system resting on an aluminium baseplate $(76.2 \times 30.5 \times 2.5 \mathrm{~cm}) .8192$ data points were sampled at $f_{s, \exp 2}=320 \mathrm{~Hz}$ in $26.5 \mathrm{~s}$ in duration. Further details may be found in [33].

A total of 17 scenarios (described in Table 3) were tested. Changing conditions were simulated either with a reduction of the stiffness of the column or by adding mass. Some scenarios involve introducing a non-linearity into the structure to simulate damage. The nonlinearities were achieved by using a bumper and a suspended column and intended to mimic a breathing crack mechanism [34] as depicted in Figure 7.

Noteworthy, the level of nonlinearity depends on the gap between the column and the bumper and the amplitude of oscillations; the results are noise-like distortions in the FRF response. The available dataset consists of 50 realisations per scenario, each one with a recorded input (expressed in terms of force time histories) and the four respective outputs (as acceleration THs, expressed in terms of [g]); therefore, in this case, 50 SIMO accelerance FRFs are at disposal for any of the 17 structural configurations. Their intended use is as Damage Sensitive Features (DSFs), as it is well-known that modal features can be exploited for SHM [35].
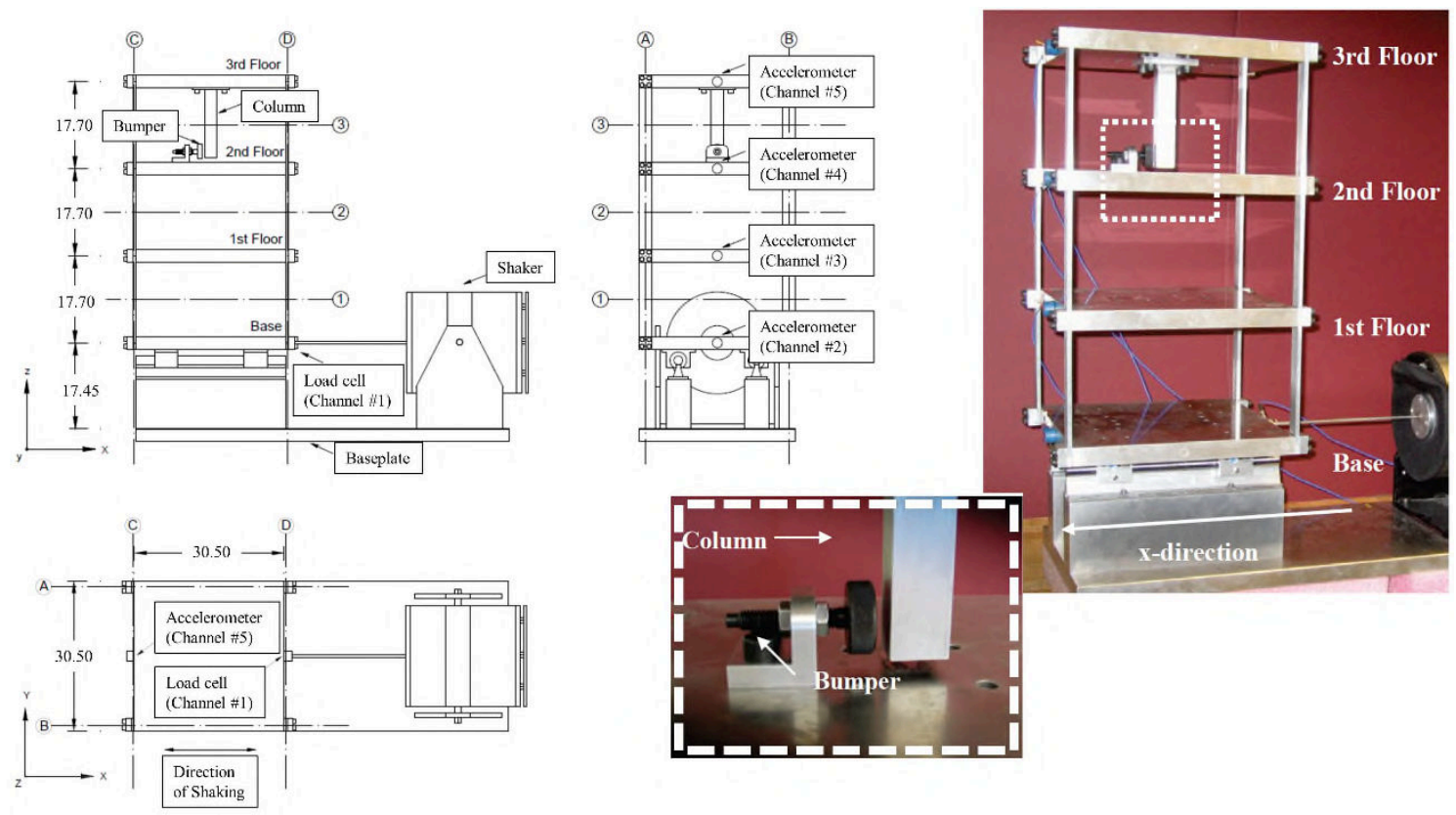

Figure 7. The experimental test setup schematics for the three-story frame structure. Rearranged from [32]. 


\begin{tabular}{|c|c|}
\hline Case & Description \\
\hline 1 & Undamaged Baseline \\
\hline 2 & Undamaged Added mass of $1.2 \mathrm{~kg}$ at the base \\
\hline 3 & Undamaged Added mass of $1.2 \mathrm{~kg}$ at the $1^{\text {st }}$ floor \\
\hline 4 & $\begin{array}{l}\text { Undamaged } 87.5 \% \text { stiffness reduction in one column of the } 1^{\text {st }} \text { inter- } \\
\text { story }\end{array}$ \\
\hline 5 & $\begin{array}{l}\text { Undamaged } 87.5 \% \text { stiffness reduction in two columns of the } 1^{\text {st }} \\
\text { inter-story }\end{array}$ \\
\hline 6 & $\begin{array}{l}\text { Undamaged } 87.5 \% \text { stiffness reduction in one column of the } 2^{\text {nd }} \\
\text { inter-story }\end{array}$ \\
\hline 7 & $\begin{array}{l}\text { Undamaged } 87.5 \% \text { stiffness reduction in one column of the } 2^{\text {nd }} \\
\text { inter-story }\end{array}$ \\
\hline 8 & $\begin{array}{l}\text { Undamaged } 87.5 \% \text { stiffness reduction in one column of the } 3^{\text {rd }} \\
\text { inter-story }\end{array}$ \\
\hline 9 & $\begin{array}{l}\text { Undamaged } 87.5 \% \text { stiffness reduction in two columns of the } 3^{\text {rd }} \\
\text { inter-story }\end{array}$ \\
\hline 10 & Damaged Distance between bumper and column tip $0.20 \mathrm{~mm}$ \\
\hline 11 & Damaged Distance between bumper and column tip $0.15 \mathrm{~mm}$ \\
\hline 12 & Damaged Distance between bumper and column tip $0.13 \mathrm{~mm}$ \\
\hline 13 & Damaged Distance between bumper and column tip $0.10 \mathrm{~mm}$ \\
\hline 14 & Damaged Distance between bumper and column tip $0.05 \mathrm{~mm}$ \\
\hline 15 & $\begin{array}{l}\text { Damaged Bumper } 0.20 \mathrm{~mm} \text { from column tip, } 1.2 \mathrm{~kg} \text { added at the } \\
\text { base }\end{array}$ \\
\hline 16 & $\begin{array}{l}\text { Damaged Bumper } 0.20 \mathrm{~mm} \text { from column tip, } 1.2 \mathrm{~kg} \text { added on the } 1^{\text {st }} \\
\text { floor }\end{array}$ \\
\hline 17 & $\begin{array}{l}\text { Damaged Bumper } 0.10 \mathrm{~mm} \text { from column tip, } 1.2 \mathrm{~kg} \text { added on the } 1^{\mathrm{st}} \\
\text { floor }\end{array}$ \\
\hline
\end{tabular}

Table 3. Damage scenarios for the experimental three-story frame structure.

\subsection{Main Case Study Experimental Results}

Figure 8 reports as an example the fitting on one acquisition for the first and the fourteenth scenarios, which represent respectively the undamaged baseline and the most nonlinear (and thus, distorted) case. All results were achieved by imposing three conjugate pairs of poles (i.e. order $\mathrm{N}=6$ ); in all cases, the RMS error was in average smaller than $4 \cdot 10^{-3}$. For brevity sake, only the results from the undamaged baseline are here described in detail; further description of all the damaged scenarios will follow. Notice that the first mode is a rigid body motion and is thus not commented. The modal parameters for scenario \#1 can be found in Table 4, compared against the values obtained through RFP and N4SID and the result retrieved from [32]. The obtained mode shapes are plotted in Figure 9.a, again compared to the results. The MAC values between this benchmark and the FRVF results can be seen in Figure 9.b; the three values are $99.1 \%$ for the first mode and $99.9 \%$ for both the second and the third mode. 
From the result in Table 4, one can notice how, in comparison with the other frequency-domain curve-fitting method (i.e. the RFP algorithm), the natural frequencies are generally slightly overestimated, while modal damping is slightly undervalued on the other hand. As always, modal damping is the most difficult to estimate and thus has the largest difference in absolute terms. Nevertheless, VF results are much closer to the ones of the time-domain method N4SID; in terms of natural frequencies, all the techniques match well with the results from [32], here assumed as the ground truth.

To conclude this discussion, a statistical study over the 50 realisations per structural case has been realised. Precisely, the mean $\mu$ and standard deviation $\sigma$ for each case have been calculated.

Figure 10 reports the boxplot diagrams for the first three natural frequencies and their respective modal damping values. One can observe that the largest uncertainty occurs for cases \#13 and \#14, i.e. the ones more affected by nonlinearity-induced distortions. Overall, the results provided by VF proved to be very stable. For convenience, the tables with the numerical values are reported in Appendix A.
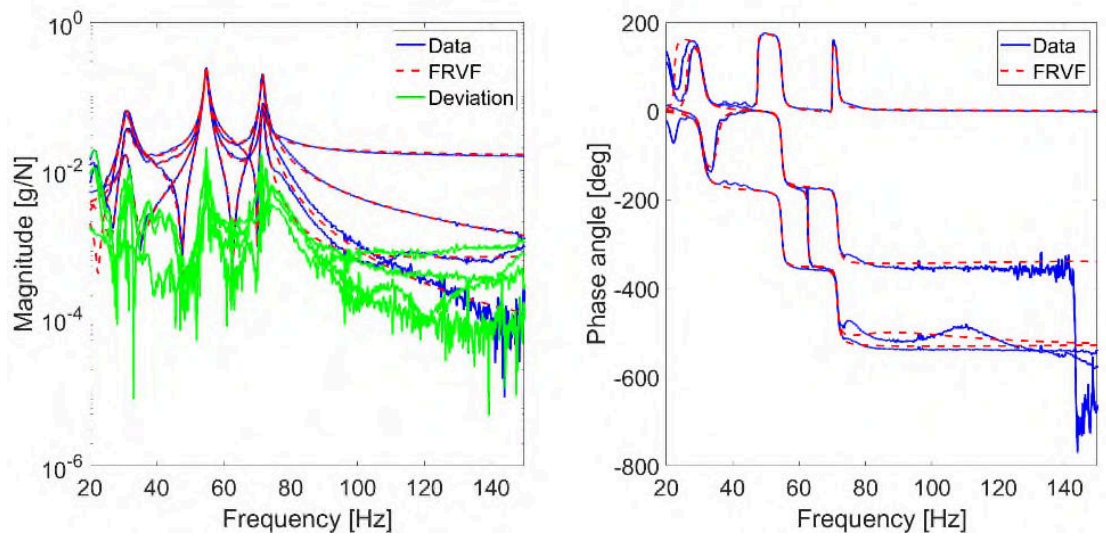

(a)
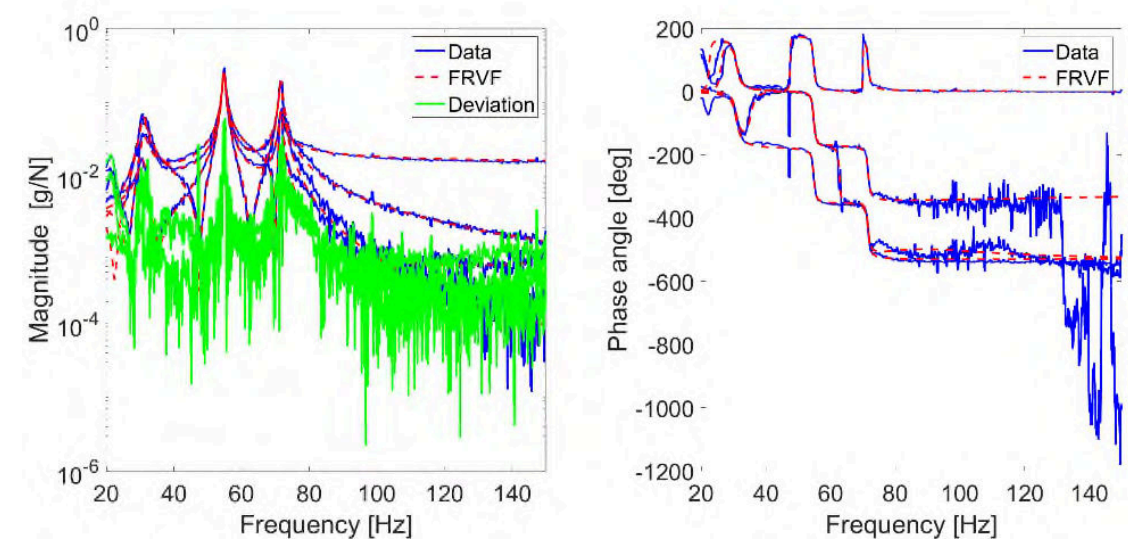

(b)

Figure 8. Three-story frame structure: Vector Fitting results: (a) case \#1 (b) case \#14. A single realisation is shown in both cases. Plot of the absolute value of gain (left) and phase angle (right). 


\begin{tabular}{|c|c|c|c|c|c|c|c|}
\hline $\begin{array}{c}\text { Mode } \\
\text { number }\end{array}$ & \multicolumn{4}{|c|}{ Frequency [Hz] } & \multicolumn{3}{|c|}{ Damping [-] } \\
\hline & VF & RFP & N4SID & $\begin{array}{c}\text { Numerical } \\
(*)\end{array}$ & VF & RFP & N4SID \\
\hline 2 & 30.89 & 30.70 & 30.89 & 29.80 & 0.035 & 0.060 & 0.048 \\
\hline 3 & 54.74 & 54.20 & 54.81 & 54.00 & 0.008 & 0.020 & 0.008 \\
\hline 4 & 71.61 & 70.70 & 71.81 & 71.60 & 0.007 & 0.009 & 0.008 \\
\hline
\end{tabular}

(*) Retrieved from [32].

Table 4. Three-story frame structure: Natural frequencies and modal damping as identified by the FRVF technique (case \#1)

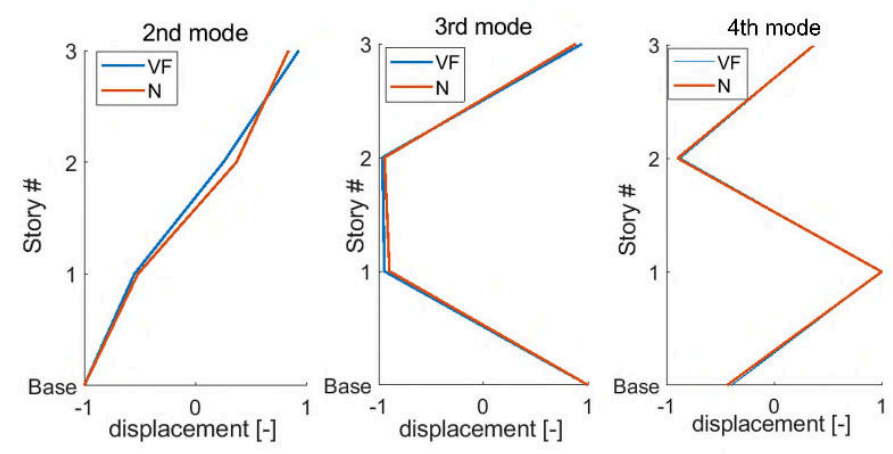

(a)

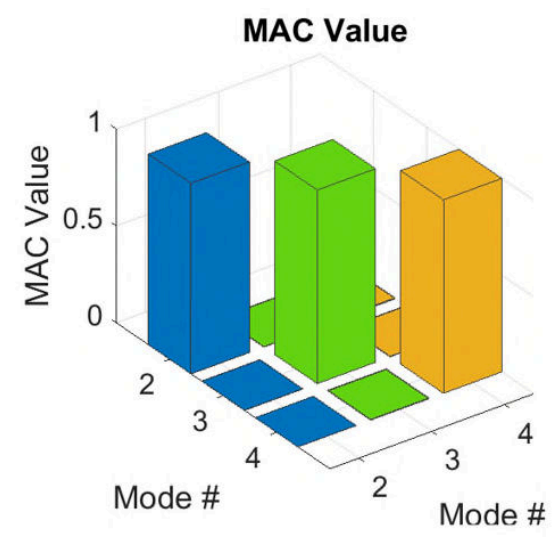

(b)

Figure 9. Three-story frame structure: (a) mode shapes of case \#1 (single realisation) superposed to the numerical results (b) MAC values between FRVF and numerical results. 


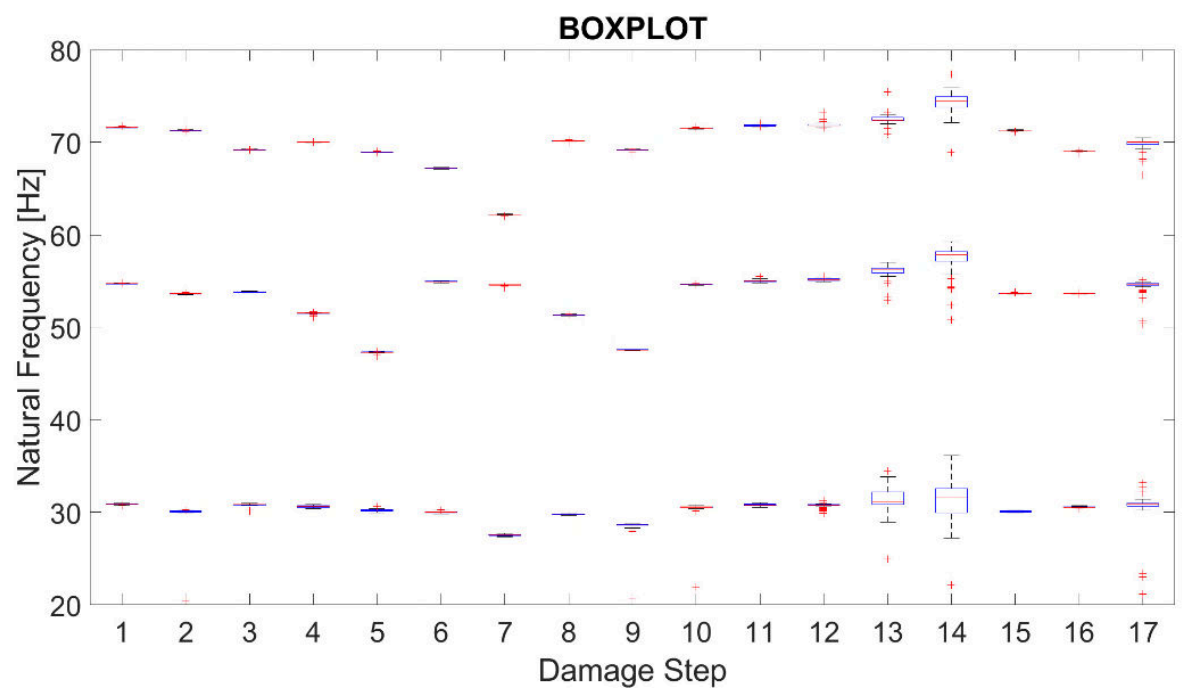

(a)

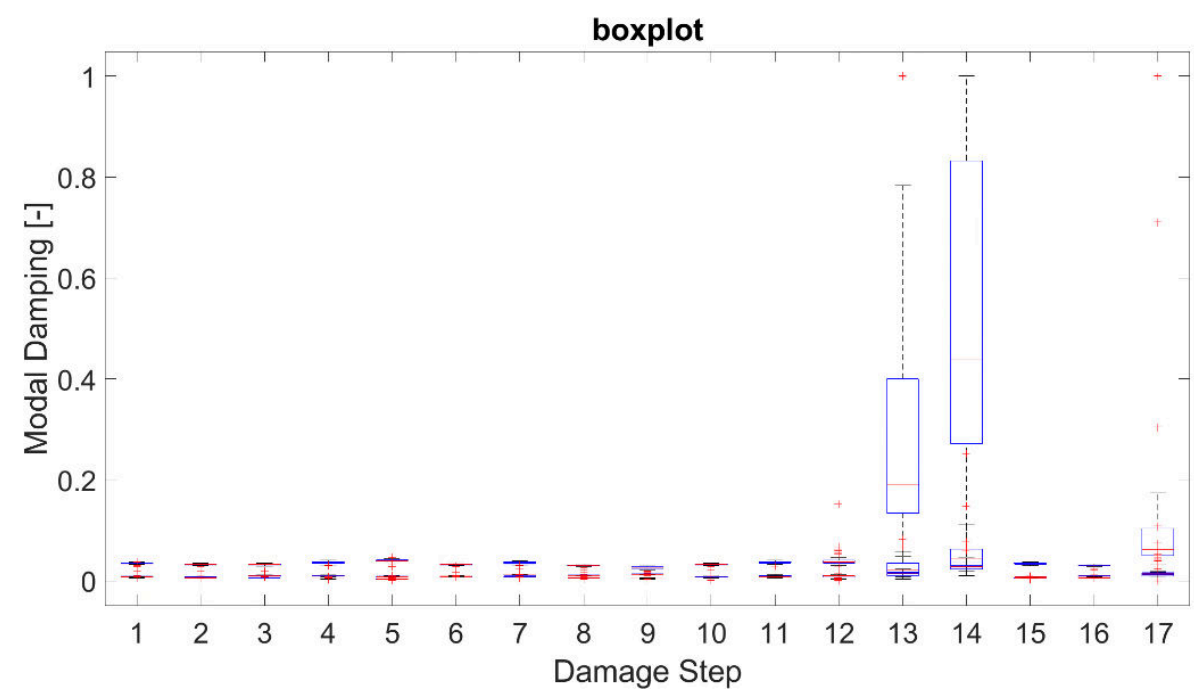

(b)

Figure 10. Three-story frame structure: Boxplot diagrams of (a) natural frequencies and (b) modal damping, all cases (50 acquisitions per case). The central red line indicates the median value, the bottom and top blue edges of the box indicate the $25^{\text {th }}$ and $75^{\text {th }}$ percentiles and the whiskers the largest and smallest data points not considered outliers. The red plus symbol indicates the outlier values.

\section{Conclusions.}

In this work, the Fast Relaxed Vector Fitting (FRVF) approach has been numerically and experimentally validated in an experimental modal analysis context.

The extraction of the mode shapes, which was never included in previous works from other fields of application, has also been added.

The proposed method can be seen as an equivalent to the Sanathanan-Koerner iteration [15], with rational basis functions (i.e. partial fractions) instead of polynomials and the implicit weighting scheme due to the pole relocation [36]. From the application of the algorithm on several numerical and experimental data of different kinds, it is possible to point out the strengths and limitations of Vector Fitting method. Firstly, the behaviour of the FRVF algorithm under emulated conditions of noisy measurement has been analysed numerically and its robustness to noise has been systematically 
investigated. This first study highlighted the following findings: (i) the proposed methodology was successful in estimating modal properties, even for high levels of noise; (ii) the estimation of modal parameters is more affected by noise presence in output signals than in the input; (iii) the modal damping is the most difficult parameter to address (as it is well-known in Literature).

After this numerical study, experimental investigations were carried out. The first case study involved the dynamic identification of a simpler structure, the spar of an aluminium prototype airwing developed in the Cranfield University laboratories. For the second case study, the same methods have been applied to a classic benchmark retrieved from Literature, a three-story frame realised in the Los Alamos National Laboratory. To simulate the changes in real-world structures caused by changing operational and environmental conditions, several sources of variability were included in this test structure. These include reducing the stiffness and adding mass at several different locations.

From these two cases, it was also possible to compare FRVF with two related and well-established SI techniques, RFP and N4SID. The RFP, which is a standard methodology for linear SI in the frequency domain, is hampered by all the typical limitations of the polynomial approximation of functions, while SSI techniques become computationally cumbersome for very large numbers of input/output channels. Therefore, the FRVF method shows evident computational advantages with respect to these similar approaches; specifically, through QR decomposition, the pole-identification step is performed efficiently and faster than for similar methods, allowing a fast fitting to the data. Overall, the modal parameters were found to be consistent.

All the results of the statistical study over the 17 structural cases and the 50 realisations per case are reported in Appendix A to provide a benchmark for comparison and the replicability of the results here obtained.

The arbitrariness of the FRVF procedure relies on the user providing the best guess of the quantity and location of the system's poles. However, by properly spacing, the location of the initial poles is only a matter of optimisation and does not represent a conceptual limit. On the other hand, the definition of the most proper model order is an actual limit, but not too different from the same issue in similar, more common techniques. Finally, an inherent limitation of the FRVF approach is that, being an inputoutput method, it may be less practical in some real uses with respect to other output-only approaches such as SSI.

\section{Acknowledgements.}

The authors wish to thank Prof. Grivet-Talocia of the Department of Electronics and Telecommunications of Politecnico di Torino for his help and precious advice.

\section{References.}

1. Richardson M, Schwarz B. Modal Parameter Estimation from Operating Data. 2003.

2. Beck JL. Bayesian system identification based on probability logic. Structural Control and Health Monitoring 2010; 17(7): 825-847. DOI: 10.1002/stc.424. 
3. Ewins DJ. Modal testing : theory, practice, and application. Research Studies Press; 2000.

4. Gustavsen B, Semlyen A. Rational approximation of frequency domain responses by vector fitting. IEEE Transactions on Power Delivery 1999; 14(3): 1052-1059. DOI: 10.1109/61.772353.

5. Deschrijver D, Mrozowski M, Dhaene T, De Zutter D. Macromodeling of multiport systems using a fast implementation of the vector fitting method. IEEE Microwave and Wireless Components Letters 2008; 18(6): 383-385. DOI: 10.1109/LMWC.2008.922585.

6. Grivet-Talocia S. The Time-Domain Vector Fitting Algorithm for Linear Macromodeling. AE $\ddot{U}$ INTERNATIONAL JOURNAL OF ELECTRONICS AND COMMUNICATIONS 2004; 58(1): 1-1. DOI: 10.1078/1434-8411-54100245.

7. Grivet-Talocia S. Package Macromodeling via Time-Domain Vector Fitting. IEEE Microwave and Wireless Components Letters 2003; 13(11): 472-474. DOI: 10.1109/LMWC.2003.819378.

8. Gustavsen B. Relaxed vector fitting algorithm for rational approximation of frequency domain responses. Proceedings - 10th IEEE Workshop on Signal Propagation on Interconnects, SPI 2006, Institute of Electrical and Electronics Engineers Inc.; 2006. DOI: 10.1109/SPI.2006.289202.

9. Gustavsen B. Improving the pole relocating properties of vector fitting. IEEE Transactions on Power Delivery 2006; 21(3): 1587-1592. DOI: 10.1109/TPWRD.2005.860281.

10. Grivet-Talocia S, Gustavsen B. Passive Macromodeling: Theory and Applications. Wiley; 2016.

11. Cabboi A, Magalhães F, Gentile C, Cunha Á. Automated modal identification and tracking: Application to an iron arch bridge. Structural Control and Health Monitoring 2017; 24(1): e1854. DOI: 10.1002/stc.1854.

12. Evans C, Rees D. Nonlinear distortions and multisine signals - Part I: measuring the best linear approximation. IEEE Transactions on Instrumentation and Measurement 2000; 49(3): 602-609. DOI: $10.1109 / 19.850402$.

13. Maia NMM, Montalvão e Silva JM (Júlio M. Theoretical and experimental modal analysis. Research Studies Press; 1997.

14. Richardson MH, Formenti DL. Global Curve Fitting of Frequency Response Measurements using the Rational Fraction Polynomial Method.

15. Sanathanan CK, Koerner J. Transfer Function Synthesis as a Ratio of Two Complex Polynomials. IEEE Transactions on Automatic Control 1963; 8(1): 56-58. DOI: 10.1109/TAC.1963.1105517.

16. Strejc V. Least squares parameter estimation. Automatica 1980; 16(5): 535-550. DOI: 10.1016/00051098(80)90077-1.

17. Semlyen A. Rational approximation of frequency domain responses by vector fitting - Power Delivery, IEEE Transactions on. vol. 14. 1999.

18. Levy EC. Complex-curve fitting. IRE Transactions on Automatic Control 2013; AC-4(1): 37-43. DOI: 10.1109/tac.1959.6429401.

19. Francis JGF. The QR Transformation A Unitary Analogue to the LR Transformation-Part 1. The Computer Journal 1961; 4(3): 265-271. DOI: 10.1093/comjn1/4.3.265.

20. Francis JGF. The QR Transformation-Part 2. The Computer Journal 1962; 4(4): 332-345. DOI: 10.1093/comjnl/4.4.332.

21. Chinea A, Grivet-Talocia S. On the parallelization of vector fitting algorithms. IEEE Transactions on Components, Packaging and Manufacturing Technology 2011; 1(11): 1761-1773. DOI: 10.1109/TCPMT.2011.2167973.

22. Van Overschee P, De Moor B. A unifying theorem for three subspace system identification algorithms. Automatica 1995; 31(12): 1853-1864. DOI: 10.1016/0005-1098(95)00072-0.

23. Verhaegen M. Identification of the deterministic part of MIMO state space models given in innovations form 
from input-output data. Automatica 1994; 30(1): 61-74. DOI: 10.1016/0005-1098(94)90229-1.

24. Ceravolo R, Asce M, Abbiati G. Time Domain Identification of Structures: Comparative Analysis of Output-Only Methods 2013. DOI: 10.1061/(ASCE)EM.1943.

25. Grivet-Talocia S, Bandinu M. Improving the convergence of vector fitting for equivalent circuit extraction from noisy frequency responses. IEEE Transactions on Electromagnetic Compatibility 2006; 48(1): 104120. DOI: $10.1109 /$ TEMC.2006.870814.

26. Pastor M, Binda M, Harčarik T. Modal assurance criterion. Procedia Engineering, vol. 48, Elsevier Ltd; 2012. DOI: $10.1016 /$ j.proeng.2012.09.551.

27. Pontillo A, Hayes D, Dussart GX, Lopez Matos GE, Carrizales MA, Yusuf SY, et al. Flexible High Aspect Ratio Wing: Low Cost Experimental Model and Computational Framework. 2018 AIAA Atmospheric Flight Mechanics Conference, Reston, Virginia: American Institute of Aeronautics and Astronautics; 2018. DOI: $10.2514 / 6.2018-1014$

28. Civera M, Zanotti Fragonara L, Surace C. Video Processing Techniques for the Contactless Investigation of Large Oscillations. Journal of Physics: Conference Series 2019; 1249(1): 012004. DOI: 10.1088/1742$6596 / 1249 / 1 / 012004$.

29. Civera M, Zanotti Fragonara L, Surace C. Using Video Processing for the Full-Field Identification of Backbone Curves in Case of Large Vibrations. Sensors 2019; 19(10): 2345. DOI: 10.3390/s19102345.

30. Civera M, Surace C, Fragonara LZ. A Computer Vision-based Approach for Non-contact Modal Analysis and Finite Element Model Updating. Under Peer Review.

31. Civera M, Ferraris M, Ceravolo R, Surace C, Betti R. The Teager-Kaiser Energy Cepstral Coefficients as an Effective Structural Health Monitoring Tool. Applied Sciences 2019; 9(23): 5064. DOI: 10.3390/app9235064.

32. Figueiredo E, Park G, Figueiras J. Structural Health Monitoring Algorithm Comparisons Using Standard Data Sets.

33. Figueiredo E, Park G, Farrar CR, Worden K, Figueiras J. Machine learning algorithms for damage detection under operational and environmental variability. Structural Health Monitoring: An International Journal 2011; 10(6): 559-572. DOI: 10.1177/1475921710388971.

34. Bovsunovsky A, Surace C. Non-linearities in the vibrations of elastic structures with a closing crack: A state of the art review. Mechanical Systems and Signal Processing 2015; 62-63: 129-148. DOI: 10.1016/j.ymssp.2015.01.021.

35. Shadan F, Khoshnoudian F, Esfandiari A. A frequency response-based structural damage identification using model updating method. Structural Control and Health Monitoring 2016; 23(2): 286-302. DOI: $10.1002 /$ stc. 1768 .

36. Hendrickx W, Deschrijver D, Dhaene T. Some Remarks on the Vector Fitting Iteration, Springer, Berlin, Heidelberg; 2006. DOI: 10.1007/3-540-28073-1_15. 
Appendix A

The values of mean and variance for the natural frequencies, modal damping, and mode shapes of the 17 cases examined in the main experimental case study are here reported.

\begin{tabular}{|c|c|c|c|c|c|c|}
\hline & \multicolumn{7}{|c|}{ FREQUENCY [Hz] } \\
\cline { 2 - 7 } & \multicolumn{2}{|c|}{$\mathbf{2}^{\text {nd }} \mathbf{m o d e}$} & \multicolumn{2}{c|}{$\mathbf{3}^{\text {rd }}$ mode } & \multicolumn{2}{c|}{$\mathbf{4}^{\text {th }}$ mode } \\
\cline { 2 - 7 } & $\boldsymbol{\mu}$ & $\boldsymbol{\sigma}$ & $\boldsymbol{\mu}$ & $\boldsymbol{\sigma}$ & $\boldsymbol{\mu}$ & $\boldsymbol{\sigma}$ \\
\hline State \#1 & 30.89 & 0.069 & 54.74 & 0.041 & 71.61 & 0.046 \\
\hline State \#2 & 30.13 & 0.1248 & 53.65 & 0.042 & 71.29 & 0.036 \\
\hline State \#3 & 30.83 & 0.108 & 53.82 & 0.048 & 69.19 & 0.035 \\
\hline State \#4 & 30.65 & 0.112 & 51.54 & 0.069 & 70.05 & 0.027 \\
\hline State \#5 & 30.25 & 0.118 & 47.30 & 0.065 & 68.92 & 0.035 \\
\hline State \#6 & 30.04 & 0.097 & 54.65 & 0.051 & 67.18 & 0.041 \\
\hline State \#7 & 27.55 & 0.074 & 54.58 & 0.047 & 62.19 & 0.043 \\
\hline State \#8 & 29.82 & 0.075 & 51.34 & 0.044 & 70.17 & 0.032 \\
\hline State \#9 & 28.80 & 0.403 & 47.56 & 0.040 & 69.16 & 0.049 \\
\hline State \#10 & 30.53 & 0.383 & 54.74 & 0.038 & 71.62 & 0.035 \\
\hline State \#11 & 30.83 & 0.112 & 55.01 & 0.113 & 71.82 & 0.078 \\
\hline State \#12 & 30.74 & 0.224 & 55.18 & 0.136 & 71.91 & 0.246 \\
\hline State \#13 & 30.70 & 4.128 & 55.78 & 1.358 & 72.38 & 0.749 \\
\hline State \#14 & 31.73 & 1.6621 & 56.98 & 1.800 & 73.71 & 1.651 \\
\hline State \#15 & 30.10 & 0.069 & 53.67 & 0.036 & 71.30 & 0.029 \\
\hline State \#16 & 30.57 & 0.068 & 53.65 & 0.035 & 69.07 & 0.032 \\
\hline State \#17 & 29.98 & 3.994 & 54.42 & 0.853 & 70.07 & 1.880 \\
\hline
\end{tabular}

\begin{tabular}{|c|c|c|c|c|c|c|}
\hline & \multicolumn{6}{|c|}{ DAMPING [-] } \\
\cline { 2 - 7 } & \multicolumn{2}{|c|}{$\mathbf{2}^{\text {nd }}$ mode } & \multicolumn{2}{c|}{$\mathbf{3}^{\text {rd }}$ mode } & \multicolumn{2}{c|}{$\mathbf{4}^{\text {th }}$ mode } \\
\cline { 2 - 7 } & $\boldsymbol{\mu}$ & $\boldsymbol{\sigma}$ & $\boldsymbol{\mu}$ & $\boldsymbol{\sigma}$ & $\boldsymbol{\mu}$ & $\boldsymbol{\sigma}$ \\
\hline State \#1 & 0.034 & 0.003 & 0.008 & $6.2 \mathrm{E}-04$ & 0.007 & $3.5 \mathrm{E}-04$ \\
\hline State \#2 & 0.032 & 0.002 & 0.007 & $7.5 \mathrm{E}-04$ & 0.006 & $4.2 \mathrm{E}-04$ \\
\hline State \#3 & 0.032 & 0.002 & 0.010 & $1.0 \mathrm{E}-03$ & 0.006 & $3.7 \mathrm{E}-04$ \\
\hline State \#4 & 0.036 & 0.002 & 0.009 & $1.1 \mathrm{E}-03$ & 0.004 & $3.6 \mathrm{E}-04$ \\
\hline State \#5 & 0.040 & 0.003 & 0.008 & $1.3 \mathrm{E}-03$ & 0.003 & $2.3 \mathrm{E}-04$ \\
\hline State \#6 & 0.032 & 0.004 & 0.008 & $3.4 \mathrm{E}-04$ & 0.009 & $4.8 \mathrm{E}-04$ \\
\hline State \#7 & 0.035 & 0.003 & 0.007 & $6.0 \mathrm{E}-04$ & 0.011 & $8.0 \mathrm{E}-04$ \\
\hline State \#8 & 0.030 & 0.003 & 0.011 & $6.8 \mathrm{E}-04$ & 0.005 & $3.7 \mathrm{E}-04$ \\
\hline State \#9 & 0.026 & 0.003 & 0.013 & $7.7 \mathrm{E}-04$ & 0.004 & $5.1 \mathrm{E}-04$ \\
\hline State \#10 & 0.031 & 0.004 & 0.008 & $8.2 \mathrm{E}-04$ & 0.007 & $9.2 \mathrm{E}-04$ \\
\hline State \#11 & 0.036 & 0.002 & 0.009 & $1.2 \mathrm{E}-03$ & 0.008 & $9.1 \mathrm{E}-04$ \\
\hline State \#12 & 0.041 & 0.018 & 0.010 & $3.2 \mathrm{E}-03$ & 0.008 & $2.4 \mathrm{E}-03$ \\
\hline State \#13 & 0.321 & 0.285 & 0.028 & $1.8 \mathrm{E}-02$ & 0.034 & $1.4 \mathrm{E}-01$ \\
\hline State \#14 & 0.530 & 0.316 & 0.055 & $4.0 \mathrm{E}-02$ & 0.030 & $1.3 \mathrm{E}-02$ \\
\hline State \#15 & 0.034 & 0.002 & 0.007 & $8.5 \mathrm{E}-04$ & 0.006 & $4.4 \mathrm{E}-04$ \\
\hline State \#16 & 0.030 & 0.002 & 0.009 & $6.0 \mathrm{E}-04$ & 0.006 & $4.0 \mathrm{E}-04$ \\
\hline State \#17 & 0.162 & 0.269 & 0.019 & $1.9 \mathrm{E}-02$ & 0.015 & $1.0 \mathrm{E}-02$ \\
\hline
\end{tabular}




\begin{tabular}{|c|c|c|c|c|c|c|}
\hline & \multicolumn{7}{|c|}{ Mode shape (base displacement) [-] } \\
\cline { 2 - 7 } & \multicolumn{2}{|c}{$\mathbf{2}^{\text {nd }}$ mode } & \multicolumn{2}{c|}{$\mathbf{3}^{\text {rd }}$ mode } & \multicolumn{2}{c|}{$\mathbf{4}^{\text {th }}$ mode } \\
\cline { 2 - 7 } & $\boldsymbol{\mu}$ & $\boldsymbol{\sigma}$ & $\boldsymbol{\mu}$ & $\boldsymbol{\sigma}$ & $\boldsymbol{\mu}$ & $\boldsymbol{\sigma}$ \\
\hline State \#1 & -1 & 0 & 1 & 0 & -0.387 & 0.006 \\
\hline State \#2 & -1 & 0 & 0.83 & 0.012 & -0.316 & 0.008 \\
\hline State \#3 & -1 & 0 & 1.00 & 0 & -0.365 & 0.012 \\
\hline State \#4 & -1 & 0 & 0.894 & 0.013 & -0.290 & 0.009 \\
\hline State \#5 & -1 & 0 & 0.732 & 0.012 & -0.175 & 0.007 \\
\hline State \#6 & -1 & 0.017 & 1 & 0 & -0.445 & 0.008 \\
\hline State \#7 & -1 & 0 & 1 & 0.005 & -0.534 & 0.010 \\
\hline State \#8 & -0.952 & 0.009 & 0.898 & 0.008 & -0.415 & 0.007 \\
\hline State \#9 & -0.798 & 0.031 & 0.854 & 0.019 & -0.449 & 0.006 \\
\hline State \#10 & -1 & 0.000 & 1 & $5.10 \mathrm{E}-04$ & -0.383 & 0.011 \\
\hline State \#11 & -1 & 0 & 1 & 0.005 & -0.400 & 0.020 \\
\hline State \#12 & -1 & 0.009 & 0.995 & 0.011 & -0.399 & 0.031 \\
\hline State \#13 & -0.989 & 0.026 & 0.925 & 0.091 & -0.410 & 0.065 \\
\hline State \#14 & -0.984 & 0.068 & 0.808 & 0.126 & -0.347 & 0.096 \\
\hline State \#15 & -1 & 0 & 0.826 & 0.011 & -0.317 & 0.009 \\
\hline State \#16 & -1 & 0.006 & 1 & 0 & -0.360 & 0.014 \\
\hline State \#17 & -0.976 & 0.038 & 0.983 & 0.059 & -0.386 & 0.073 \\
\hline
\end{tabular}

\begin{tabular}{|c|c|c|c|c|c|c|}
\hline & \multicolumn{6}{|c|}{ Mode shape (1 $\mathbf{1}^{\circ}$ floor displacement) [-] } \\
\cline { 2 - 7 } & \multicolumn{2}{|c|}{$\mathbf{2}^{\text {nd }}$ mode } & \multicolumn{2}{c|}{$\mathbf{3}^{\text {rd }}$ mode } & \multicolumn{2}{c|}{$\mathbf{4}^{\text {th }}$ mode } \\
\cline { 2 - 7 } & $\boldsymbol{\mu}$ & $\boldsymbol{\sigma}$ & $\boldsymbol{\mu}$ & $\boldsymbol{\sigma}$ & $\boldsymbol{\mu}$ & $\boldsymbol{\sigma}$ \\
\hline State \#1 & -0.540 & 0.006 & -0.953 & 0.009 & 1 & 0 \\
\hline State \#2 & -0.491 & 0.008 & -1 & 0 & 1 & 0 \\
\hline State \#3 & -0.556 & 0.008 & -0.853 & 0.010 & 0.867 & 0.013 \\
\hline State \#4 & -0.475 & 0.014 & -1 & 0 & 0.925 & 0.016 \\
\hline State \#5 & -0.370 & 0.012 & -1 & 0 & 0.747 & 0.017 \\
\hline State \#6 & -0.632 & 0.009 & -0.889 & 0.010 & 1 & 0 \\
\hline State \#7 & -0.723 & 0.009 & -0.941 & 0.013 & 1 & 0 \\
\hline State \#8 & -0.552 & 0.009 & -0.651 & 0.010 & 1 & 0 \\
\hline State \#9 & -0.543 & 0.015 & -0.389 & 0.012 & 1 & 0 \\
\hline State \#10 & -0.519 & 0.049 & -0.969 & 0.021 & 1 & 0 \\
\hline State \#11 & -0.522 & 0.013 & -0.969 & 0.026 & 1 & 0 \\
\hline State \#12 & -0.507 & 0.026 & -0.968 & 0.037 & 1 & 0 \\
\hline State \#13 & -0.487 & 0.196 & -0.979 & 0.061 & 0.974 & 0.075 \\
\hline State \#14 & -0.412 & 0.404 & -0.999 & 0.006 & 0.860 & 0.266 \\
\hline State \#15 & -0.490 & 0.006 & -1 & 0 & 1 & 0 \\
\hline State \#16 & -0.542 & 0.009 & -0.872 & 0.015 & 0.867 & 0.014 \\
\hline State \#17 & -0.467 & 0.206 & -0.895 & 0.052 & 0.822 & 0.205 \\
\hline
\end{tabular}




\begin{tabular}{|c|c|c|c|c|c|c|}
\hline & \multicolumn{6}{|c|}{ Mode shape $\left(2^{\circ}\right.$ floor displacement) $[-]$} \\
\hline & \multicolumn{2}{|c|}{$2^{\text {nd }}$ mode } & \multicolumn{2}{|c|}{$3^{\text {rd }}$ mode } & \multicolumn{2}{|c|}{$4^{\text {th }}$ mode } \\
\hline & $\mu$ & $\sigma$ & $\mu$ & $\sigma$ & $\mu$ & $\sigma$ \\
\hline State \#1 & 0.245 & 0.006 & -0.946 & 0.008 & -0.883 & 0.009 \\
\hline State \#2 & 0.295 & 0.016 & -0.858 & 0.008 & -0.918 & 0.012 \\
\hline State \#3 & 0.270 & 0.006 & -0.724 & 0.006 & -1 & 0 \\
\hline State \#4 & 0.225 & 0.007 & -0.672 & 0.006 & -1 & 0 \\
\hline State \#5 & 0.202 & 0.011 & -0.441 & 0.005 & -1 & 0 \\
\hline State \#6 & 0.284 & 0.009 & -0.939 & 0.008 & -0.836 & 0.015 \\
\hline State \#7 & 0.432 & 0.015 & -0.976 & 0.019 & -0.784 & 0.018 \\
\hline State \#8 & 0.179 & 0.007 & -1 & 0 & -0.710 & 0.006 \\
\hline State \#9 & 0.032 & 0.017 & -1 & 0 & -0.595 & 0.006 \\
\hline State \#10 & 0.270 & 0.051 & -0.944 & 0.016 & -0.885 & 0.019 \\
\hline State \#11 & 0.258 & 0.010 & -0.925 & 0.016 & -0.880 & 0.022 \\
\hline State \#12 & 0.272 & 0.019 & -0.894 & 0.032 & -0.871 & 0.046 \\
\hline State \#13 & 0.310 & 0.051 & -0.775 & 0.092 & -0.897 & 0.076 \\
\hline State \#14 & 0.356 & 0.074 & -0.613 & 0.106 & -0.932 & 0.283 \\
\hline State \#15 & 0.300 & 0.008 & -0.856 & 0.010 & -0.909 & 0.021 \\
\hline State \#16 & 0.288 & 0.006 & -0.723 & 0.010 & -1 & 0 \\
\hline State \#17 & 0.305 & 0.032 & -0.643 & 0.042 & -0.960 & 0.283 \\
\hline
\end{tabular}

\begin{tabular}{|c|c|c|c|c|c|c|}
\hline & \multicolumn{6}{|c|}{ Mode shape (3 ${ }^{\circ}$ floor displacement) [-] } \\
\cline { 2 - 7 } & \multicolumn{2}{|c|}{$\mathbf{2}^{\text {nd }}$ mode } & \multicolumn{2}{|c|}{$\mathbf{3}^{\text {rd }}$ mode } & \multicolumn{2}{c|}{$\mathbf{4}^{\text {th }}$ mode } \\
\cline { 2 - 7 } & $\boldsymbol{\mu}$ & $\boldsymbol{\sigma}$ & $\boldsymbol{\mu}$ & $\boldsymbol{\sigma}$ & $\boldsymbol{\mu}$ & $\boldsymbol{\sigma}$ \\
\hline State \#1 & 0.926 & 0.009 & 0.927 & 0.009 & 0.383 & 0.006 \\
\hline State \#2 & 0.955 & 0.027 & 0.903 & 0.012 & 0.402 & 0.006 \\
\hline State \#3 & 0.994 & 0.007 & 0.776 & 0.008 & 0.449 & 0.005 \\
\hline State \#4 & 0.835 & 0.024 & 0.807 & 0.007 & 0.464 & 0.006 \\
\hline State \#5 & 0.689 & 0.006 & 0.735 & 0.010 & 0.489 & 0.010 \\
\hline State \#6 & 0.886 & 0.021 & 0.919 & 0.009 & 0.367 & 0.007 \\
\hline State \#7 & 0.859 & 0.011 & 0.991 & 0.015 & 0.386 & 0.016 \\
\hline State \#8 & 1 & 0 & 0.821 & 0.006 & 0.237 & 0.003 \\
\hline State \#9 & 0.998 & 0.015 & 0.645 & 0.007 & 0.141 & 0.004 \\
\hline State \#10 & 0.933 & 0.019 & 0.938 & 0.015 & 0.386 & 0.016 \\
\hline State \#11 & 0.923 & 0.011 & 0.923 & 0.018 & 0.398 & 0.015 \\
\hline State \#12 & 0.931 & 0.028 & 0.915 & 0.025 & 0.393 & 0.036 \\
\hline State \#13 & 0.860 & 0.233 & 0.852 & 0.250 & 0.441 & 0.057 \\
\hline State \#14 & 0.710 & 0.336 & 0.841 & 0.066 & 0.542 & 0.229 \\
\hline State \#15 & 0.957 & 0.008 & 0.900 & 0.011 & 0.403 & 0.010 \\
\hline State \#16 & 0.996 & 0.005 & 0.789 & 0.011 & 0.447 & 0.007 \\
\hline State \#17 & 0.935 & 0.183 & 0.770 & 0.044 & 0.442 & 0.241 \\
\hline
\end{tabular}


2021-01-06

\title{
Experimental modal analysis of
}

\section{structural systems by using the fast relaxed vector fitting method}

\author{
Civera, Marco
}

Wiley

Civera M, Calamei G, Zanotti Fragonara L. (2021) Experimental modal analysis of structural systems by using the fast relaxed vector fitting method. Structural Control and Health Monitoring, Volume 28, Issue 4, April 2021, Article number e2695

https://doi.org/10.1002/stc.2695

Downloaded from Cranfield Library Services E-Repository 